\title{
Absorbing aerosol radiative effects in the limb-scatter viewing geometry
}

\author{
A. Wiacek ${ }^{1,2,3}$, R. V. Martin ${ }^{1,5}$, A. E. Bourassa ${ }^{4}$, N. D. Lloyd ${ }^{4}$, and D. A. Degenstein ${ }^{4}$ \\ ${ }^{1}$ Department of Physics and Atmospheric Science, Dalhousie University, Halifax, Canada \\ ${ }^{2}$ Department of Environmental Science, Saint Mary's University, Halifax, Nova Scotia, Canada \\ ${ }^{3}$ Department of Astronomy \& Physics, Saint Mary's University, Halifax, Nova Scotia, Canada \\ ${ }^{4}$ Department of Physics and Engineering Physics, University of Saskatchewan, Saskatoon, Canada \\ ${ }^{5}$ Harvard-Smithsonian Center for Astrophysics, Cambridge, Massachusetts, USA
}

Correspondence to: A. Wiacek (aldona.wiacek@smu.ca)

Received: 13 January 2013 - Published in Atmos. Meas. Tech. Discuss.: 19 February 2013

Revised: 22 August 2013 - Accepted: 3 September 2013 - Published: 22 October 2013

\begin{abstract}
The limb-scatter satellite viewing geometry is well suited to detecting low-concentration aerosols in the upper troposphere due to its long observation path length $(\sim 200 \mathrm{~km})$, high vertical resolution $(\sim 1-2 \mathrm{~km})$ and good geographic coverage. We use the fully three-dimensional radiative transfer code SASKTRAN to simulate the sensitivity of limb-scatter viewing Odin/OSIRIS satellite measurements to absorbing mineral dust and carbonaceous aerosols (smoke and pure soot), as well as to non-absorbing sulfate aerosols and ice in the upper troposphere.

At long wavelengths $(813 \mathrm{~nm})$ the addition of all aerosols (except soot) to an air only atmosphere produced a radiance increase as compared to air only, on account of the low Rayleigh scattering in air only at $813 \mathrm{~nm}$. The radiance reduction due to soot aerosol was negligible $(<0.1 \%)$ at all heights $(0-100 \mathrm{~km})$.

At short wavelengths $(337,377,452 \mathrm{~nm})$, we found that the addition of any aerosol species to an air only atmosphere caused a decrease in single-scattered radiation due to an extinction of Rayleigh scattering in the direction of OSIRIS. The reduction was clearly related to particle size first, with absorption responsible for second-order effects only. Multiple-scattered radiation could either increase or decrease in the presence of an aerosol species, depending both on particle size and absorption. Large scatterers (ice, mineral dust) all increased multiple-scattered radiation within, below and above the aerosol layer. Small, highly absorbing pure soot particles produced a negligible multiple-scattering response $(<0.1 \%)$ at all heights, primarily confined to within
\end{abstract}

and below the soot layer. Medium-sized scatterers produced a multiple-scattering response that depended on their absorbing properties. Increased radiances were simulated as compared to air only at all short wavelengths $(337,377$ and $452 \mathrm{~nm}$ ) for sulfate aerosol particles (non-absorbing) while decreased radiances were simulated for smoke particles (absorbing) at 337 and $377 \mathrm{~nm}$, where multiple scattering involving the medium-sized carbonaceous particles amplified their absorbing properties. At $452 \mathrm{~nm}$, however, this effect was attenuated and albedo-dependent.

At short wavelengths, the combined effect of single scattering decreases and multiple scattering increases led to complex total radiance signatures that generally could not unambiguously distinguish absorbing versus non-absorbing aerosols. Smoke aerosols led to a total radiance decrease (as compared to air only) at all altitudes above the aerosol layer $(15-100 \mathrm{~km})$. This unique signature was a result of the aerosols' strong absorbing properties, non-negligible scattering efficiency, and number concentrations high enough to make multiple scattering effects due to the aerosol itself significant. Thus, in the limb-scatter viewing geometry scene darkening above the aerosol layer is unambiguously due to absorption whereas scene darkening within and below the aerosol layer can simply be the result of a reduction in single-scattered radiance. Our simulations show a greater scene darkening for decreasing wavelengths, increasing surface albedo, decreasing solar zenith angle, and increasing particle number concentration, however, at $337 \mathrm{~nm}$ this effect did not exceed $0.5 \%$ of the total radiance due to air 
only, making the unique identification of medium-sized carbonaceous aerosols, i.e., smoke, difficult. Scene darkening (or brightening) varies linearly with particle number concentration over three orders of magnitude.

A fortuitous, unexpected implication of our analysis is that limb-scatter retrievals of aerosol extinction are not sensitive to external information about aerosol absorption.

\section{Introduction}

Atmospheric aerosol particles in the upper troposphere cause a strong climate forcing via their direct and indirect radiative effects. Considerable uncertainty in these radiative effects arises from the paucity of observations of chemical composition and spatial distribution of aerosols in the upper troposphere/lower stratosphere (UTLS) region. At present, observations of upper tropospheric aerosol are sparse, coming primarily from infrequent aircraft campaigns. Advances in satellite remote sensing could yield global 3-D observations of atmospheric aerosols in the UTLS.

The limb-scatter satellite viewing geometry is well suited to detecting low-concentration aerosols in the upper troposphere due to its long observation path length $(\sim 200 \mathrm{~km})$, high vertical resolution $(\sim 1-2 \mathrm{~km})$ and good geographic coverage. A possible complication in a retrieval of aerosol extinction in the UTLS is the need for a priori information on aerosol single scattering albedo. Thus, it is important to characterize the signal of absorbing aerosols (mineral dust and carbonaceous aerosols) in limb-scatter satellite observations and to compare it to the signal arising from non-absorbing aerosols (sulfate and ice). Such an analysis also contributes to the goal of inferring aerosol composition from limb-scatter satellite observations.

Past, present and upcoming measurements of UTLS aerosols from satellite-based platforms in limb-viewing mode are summarized in Table 1 and include both direct solar/stellar occultation and limb-scattered sunlight measurements. Most retrievals have used occultation techniques with sparse coverage and have focused on the lower stratosphere, where aerosol composition is more homogeneous. Of instruments capable of UV/Vis measurements in limb-scattered viewing mode, only OSIRIS and OMPS are at present operational, with OSIRIS providing the longest observational record since 2001. The suitability of SCIAMACHY limbviewing measurements for deriving stratospheric aerosol extinction has recently been demonstrated (Taha et al., 2011; Ernst et al., 2012). Finally, OSIRIS measurements of limbscattered sunlight have been used to derive subvisual cirrus frequency of occurrence and cloud optical depth in the UTLS (Bourassa et al., 2005), volcanic stratospheric aerosol concentrations (Bourassa et al., 2010) and their climatic effects (Kravitz et al., 2010, 2011; Haywood et al., 2010), as well as ozone and other trace gas vertical profiles such as
$\mathrm{NO}_{2}$ and $\mathrm{BrO}$ (Haley et al., 2004; Degenstein et al., 2009; Bourassa et al., 2011; McLinden et al., 2010). Moreover, Bourassa et al. (2007) present a retrieval of stratospheric aerosol number concentration assuming a particle size distribution. A retrieval of stratospheric aerosol size information by incorporating the $1.5 \mu \mathrm{m}$ channel information is described by Bourassa et al. (2008a).

Of the satellite-based measurements discussed above, little attention has been devoted to the retrieval of aerosol extinction in the upper troposphere. None have reported attempts to discriminate aerosol composition. We note that significant work on aerosol typing, especially in the lower troposphere, has been performed by the CALIPSO satellite team, which uses the depolarization and color ratio of a 532 and $1064 \mathrm{~nm}$ laser together with season, altitude and signal threshold to infer aerosol type (Omar et al., 2009). In this study, we use the fully three-dimensional radiative transfer code SASKTRAN (Bourassa et al., 2008b), to simulate the sensitivity of limb-scatter viewing Odin/OSIRIS measurements to upper tropospheric absorbing and non-absorbing aerosols.

\section{Methodology}

The radiative transfer calculations in our study are carried out using SASKTRAN, which is a $\mathrm{C}++$ code optimized to run efficiently on a multi-threaded desktop and Linux platforms. SASKTRAN evaluates the scalar equation of radiative transfer with multiple scattering while employing a fully threedimensional, spherical shell model of the atmosphere. The atmospheric model within SASKTRAN incorporates scattering by air molecules and aerosols with altitude-dependent cross-sections and phase functions, which can be either calculated or specified directly. The aerosol scatterers can additionally be given absorbing properties via their index of refraction. Temperature-dependent absorption can be specified for an expandable list of gaseous species, e.g., $\mathrm{O}_{3}$ and $\mathrm{NO}_{2}$. Scattering is traced exactly for first and second order scattering events using the successive orders of scattering approach; higher-order scattering term contributions at a given point along the OSIRIS line-of-sight are estimated by integrating the previous order scattering source term at the local zenith. Reflection at the Earth's surface is modeled as Lambertian. Details of the method and implementation are discussed by Bourassa et al. (2008b).

All simulations discussed below were performed at two albedos, $a=[0,1]$, four wavelengths, $\lambda=[337,377,452$, $813 \mathrm{~nm}]$, and two scan geometries. OSIRIS scan 34196006 corresponds to the lowest observation solar zenith angle for OSIRIS (SZA $=\sim 65^{\circ}$ ), a latitude of $\sim 70^{\circ}$, a longitude of $\sim 170^{\circ}$, and an observation solar azimuth angle (SAA) of $\sim 70^{\circ}$. Scan 33736001 corresponds to a high SZA $\left(\sim 87^{\circ}\right)$, a latitude of $\sim 35^{\circ}$, a longitude of $\sim 111^{\circ}$, and an SAA of $\sim 64^{\circ}$, which is very similar to that of the low-SZA scan. The solar scattering angle of the two scans is similar and $\sim 68^{\circ}$, 
Table 1. Satellite retrievals of aerosol extinction in the UTLS using the limb-viewing technique.

\begin{tabular}{|c|c|c|c|c|}
\hline Instrument & Geometry & Coverage & Channels & Technique \\
\hline HALOE & Solar occultation & $1991-2005$ & $\begin{array}{l}5 \text { gas channels } \\
(2.45-5.26 \mu \mathrm{m}) \text { also used for aerosols }\end{array}$ & $\begin{array}{l}\text { gas filter and broad- } \\
\text { band radiometer }\end{array}$ \\
\hline POAM III & Solar occultation & $1998-2005$ & $\begin{array}{l}9 \text { channels ( } 353-1060 \mathrm{~nm} \text { ) including } 7 \text { for gases and } 6 \\
\text { for aerosols (with functional overlap) }\end{array}$ & photometer \\
\hline SAM II & Solar occultation & $1978-1993$ & 1 channel for aerosols at $1.0 \mu \mathrm{m}(0.038 \mu \mathrm{m}$ bandwidth $)$ & photometer \\
\hline SAGE I & Solar occultation & $1979-1982$ & 4 channels $(385-1000 \mathrm{~nm})$ for aerosols and $\mathrm{O}_{3}, \mathrm{NO}_{2}$ & photometer \\
\hline SAGE II & Solar occultation & $1984-2005$ & 7 channels $(385-1020 \mathrm{~nm})$ for aerosols, $\mathrm{O}_{3}, \mathrm{NO}_{2}, \mathrm{H}_{2} \mathrm{O}$ & photometer \\
\hline SAGE III* & $\begin{array}{l}\text { Solar occultation and } \\
\text { limb-scattered sunlight }\end{array}$ & $2001-2006$ & $290-1030 \mathrm{~nm}$ & grating spectrometer \\
\hline OSIRIS* & Limb-scattered sunlight & $2001-$ & $270-810 \mathrm{~nm}$ & grating spectrometer \\
\hline SCIAMACHY & Limb-scattered sunlight & $2002-2012$ & $\begin{array}{l}240-314 \mathrm{~nm}, 309-405 \mathrm{~nm} \\
394-620 \mathrm{~nm}, 604-805 \mathrm{~nm} \\
785-1050 \mathrm{~nm}, 1000-1750 \mathrm{~nm}\end{array}$ & grating spectrometer \\
\hline GOMOS** & Stellar occultation & $2002-2012$ & $\begin{array}{l}248-371 / 387-693 \mathrm{~nm} \\
750-776 \mathrm{~nm} \\
915-956 \mathrm{~nm}\end{array}$ & grating spectrometer \\
\hline ACE-FTS & Solar occultation & $2004-$ & $750-4400 \mathrm{~cm}^{-1}$ (mid-IR) & FTIR spectrometer \\
\hline OMPS & Limb-scattered sunlight & $2011-$ & $290-1000 \mathrm{~nm}$ & grating spectrometer \\
\hline
\end{tabular}

allowing us to compare the effect of increasing observation SZA on sensitivity to UTLS aerosols.

We performed an analysis of single, multiple, and total limb-scattered radiance signatures arising from gradually adding components to the model atmosphere as follows: (1) simulate air molecules alone (no absorbing gaseous species or scattering/absorbing aerosol species), (2) simulate a given aerosol component alone, (3) simulate air molecules together with the single aerosol component. The radiances are broken down into single scattering and multiple scattering components, the latter obtained from taking the difference between a SASKTRAN calculation performed for 50 scattering orders and a single scattering calculation. We examine multiple-scattered radiation separately from singlescattered radiation to better understand the vertical distribution of the change in scattered signal at OSIRIS due to the addition of an aerosol layer. Furthermore, this separation into single- and multiple-scattered radiation gives us additional insight into the relative role of absorption and scattering in the limb-viewing geometry.

The single scattering and multiple scattering components both include aerosol absorption as specified via its complex refractive index. In all cases that an aerosol layer was simulated, it was placed in a $5 \mathrm{~km}$ layer between $10-15 \mathrm{~km}$ in the atmosphere, at some number concentration, $\rho$. The aerosol layers were specified with a lognormal size distribution with the properties shown in Table 2. Air molecules, when simulated, occupied the entire model atmosphere from
0-100 km. Our simulations included the same set of tangent heights, regardless of which species were present in the model atmosphere.

\subsection{Mineral dust aerosol properties}

Mineral dust aerosol was modeled as comprising lognormally distributed spherical particles with a realistic mode radius $(\sim 1 \mu \mathrm{m})$ and mode width $(\sim 1.6 \mu \mathrm{m})$ following Tegen (2003). The number concentration of $0.2 \mathrm{~cm}^{-3}$ in the aerosol layer represents a 20 -fold dust enhancement above the typical free-tropospheric background of ice-active nuclei of $\sim 0.01 \mathrm{~cm}^{-3}$ (DeMott et al., 2003). While ice nuclei concentrations between 1 and $20 \mathrm{~cm}^{-3}$ have been measured in the boundary layer near dust emission sources (Ansmann et al., 2008), such high concentrations are not realistic to use in simulations involving the UTLS region. As shown by Wiacek at al. (2010), most mineral dust will have undergone cloud-processing and washout before reaching the upper troposphere, in addition to dilution caused by the expansion of rising air parcels.

In order to separate the effects of absorption and scattering, an absorbing (D1) and a non-absorbing (D2) dust were simulated separately. The detailed scattering and absorption characteristics are given in Table 3. The optical thickness of the $5 \mathrm{~km}$ dust layer at an SZA of $\sim 65^{\circ}(\sim 2 \mathrm{X}$ path length) and at an SZA of $\sim 87^{\circ}(\sim 20 \mathrm{X}$ path length) corresponds to the two scan geometries in our study, with vertical optical thickness in the $5 \mathrm{~km}$ layer also shown. The 
Table 2. Physical (number concentration $\rho$, lognormal mode radius, $r$, lognormal mode width, $\sigma$ ) and optical (complex index of refraction $n_{\mathrm{r}}, n_{\mathrm{i}}$ ) properties of aerosol layers simulated either alone (between 10-15 km) or in conjunction with air molecules (between 0-100 km).

\begin{tabular}{|c|c|c|c|c|c|c|c|c|c|c|}
\hline \multirow[b]{2}{*}{ Aerosol } & \multirow{2}{*}{$\begin{array}{r}\rho \\
{\left[\mathrm{cm}^{-3}\right]}\end{array}$} & \multirow{2}{*}{$\begin{array}{c}r, \sigma \\
{[\mu \mathrm{m}]}\end{array}$} & \multicolumn{4}{|c|}{$n_{\mathrm{r}}$} & \multicolumn{4}{|c|}{$n_{\mathrm{i}}$} \\
\hline & & & $337 \mathrm{~nm}$ & $377 \mathrm{~nm}$ & $452 \mathrm{~nm}$ & $813 \mathrm{~nm}$ & $337 \mathrm{~nm}$ & $377 \mathrm{~nm}$ & $452 \mathrm{~nm}$ & $813 \mathrm{~nm}$ \\
\hline Absorbing dust (D1) & 0.2 & $1.0,1.6$ & 1.55 & 1.55 & 1.55 & 1.55 & $1.953 \times 10^{-2}$ & $1.380 \times 10^{-2}$ & $6.928 \times 10^{-3}$ & $6.441 \times 10^{-4}$ \\
\hline Non-absorbing dust (D2) & 0.2 & $1.0,1.6$ & 1.55 & 1.55 & 1.55 & 1.55 & 0 & 0 & 0 & 0 \\
\hline Pure soot (PS) & 20 & $0.0125,1.6$ & 1.95 & 1.95 & 1.95 & 1.95 & 0.79 & 0.79 & 0.79 & 0.79 \\
\hline Smoke (SM) & 20 & $0.075,1.6$ & 1.51 & 1.51 & 1.51 & 1.53 & 0.1157 & 0.0890 & $2.677 \times 10^{-2}$ & $1.653 \times 10^{-2}$ \\
\hline Sulfate aerosol (SO4) & 30 & $0.08,1.6$ & 1.47 & 1.45 & 1.43 & 1.43 & $1.0 \times 10^{-10}$ & $2.1 \times 10^{-10}$ & $2.5 \times 10^{-9}$ & $8.8 \times 10^{-8}$ \\
\hline Ice (ICE) & 0.2 & $1.0,1.6$ & 1.33 & 1.32 & 1.32 & 1.30 & $5.5 \times 10^{-9}$ & $2.7 \times 10^{-9}$ & $1.7 \times 10^{-9}$ & $1.5 \times 10^{-7}$ \\
\hline
\end{tabular}

Table 3. Scattering, absorption and extinction cross-sections $\left(k_{\mathrm{s}}, k_{\mathrm{a}}, k_{\mathrm{e}}\right.$, respectively) of absorbing dust (D1) and the scattering cross-section of non-absorbing dust (D2) as a function of wavelength $(\lambda)$. The single scatter albedo ( $\omega$, unitless) of D1 is also shown. Note that the scattering optical thickness of D2 is equivalent to the extinction optical thickness of D1. The scattering, absorption and extinction optical thickness $\left(\tau_{\mathrm{s}}, \tau_{\mathrm{a}}, \tau_{\mathrm{e}}\right.$, respectively, unitless) is shown for a $5 \mathrm{~km}$ layer of D1, as described in Table 2, under three viewing conditions.

\begin{tabular}{|c|c|c|c|c|c|c|c|c|c|c|c|c|c|c|}
\hline \multirow[b]{2}{*}{$\lambda[\mathrm{nm}]$} & \multicolumn{4}{|c|}{ D1 } & \multirow{2}{*}{$\begin{array}{r}\mathrm{D} 2 \\
k_{\mathrm{s}}\left[\mathrm{cm}^{2}\right]\end{array}$} & \multicolumn{3}{|c|}{$5 \mathrm{~km}$ layer (vertical) } & \multicolumn{3}{|c|}{$5 \mathrm{~km}$ layer $\left(\mathrm{SZA}=65^{\circ}\right)$} & \multicolumn{3}{|c|}{$5 \mathrm{~km}$ layer $\left(\mathrm{SZA}=87^{\circ}\right)$} \\
\hline & $k_{\mathrm{s}}\left[\mathrm{cm}^{2}\right]$ & $k_{\mathrm{a}}\left[\mathrm{cm}^{2}\right]$ & $k_{\mathrm{e}}\left[\mathrm{cm}^{2}\right]$ & $\omega$ & & $\tau_{\mathrm{S}}$ & $\tau_{\mathrm{a}}$ & $\tau_{\mathrm{e}}$ & $\tau_{\mathrm{s}}$ & $\tau_{\mathrm{a}}$ & $\tau_{\mathrm{e}}$ & $\tau_{\mathrm{S}}$ & $\tau_{\mathrm{a}}$ & $\tau_{\mathrm{e}}$ \\
\hline 337 & $6.46 \times 10^{-8}$ & $4.37 \times 10^{-8}$ & $1.08 \times 10^{-7}$ & 0.60 & $1.08 \times 10^{-7}$ & 0.0065 & 0.0044 & 0.0108 & 0.0153 & 0.0104 & 0.0256 & 0.1235 & 0.0836 & 0.2071 \\
\hline 377 & $7.14 \times 10^{-8}$ & $3.79 \times 10^{-8}$ & $1.09 \times 10^{-7}$ & 0.65 & $1.09 \times 10^{-7}$ & 0.0071 & 0.0038 & 0.0109 & 0.0169 & 0.0090 & 0.0258 & 0.1363 & 0.0724 & 0.2087 \\
\hline 452 & $8.65 \times 10^{-8}$ & $2.43 \times 10^{-8}$ & $1.11 \times 10^{-7}$ & 0.78 & $1.11 \times 10^{-7}$ & 0.0087 & 0.0024 & 0.0111 & 0.0205 & 0.0057 & 0.0262 & 0.1653 & 0.0464 & 0.2117 \\
\hline 813 & $1.15 \times 10^{-7}$ & $2.23 \times 10^{-9}$ & $1.18 \times 10^{-7}$ & 0.98 & $1.18 \times 10^{-7}$ & 0.0115 & 0.0002 & 0.0118 & 0.0273 & 0.0005 & 0.0278 & 0.2205 & 0.0043 & 0.2248 \\
\hline
\end{tabular}

real index of refraction of mineral dust was set to $n_{\mathrm{r}}=1.55$ at all wavelengths, which is in line with the work of Sokolik and Toon (1999). The imaginary index of refraction of mineral dust is based on the field measurements of Müller et al. (2009) and includes a steep decrease in absorption towards visible and infrared wavelengths (Table 2). Our choice of relatively absorbing and large mineral dust particles leads to lower single scatter albedo values than those reported in the literature for AERONET and other ground-based techniques (Müller et al., 2012). These low single scattering albedos allow assessment of an upper limit of aerosol absorption. Finally, we carried out radiative transfer calculations with identically absorbing and similarly distributed mixtures of spheroid particles and found the effects of particle non-sphericity to be small.

\subsection{Carbonaceous aerosol properties}

Carbonaceous aerosol particle properties were modeled as either highly absorbing small particles (pure soot) or less absorbing bigger particles (smoke). In the case of pure soot, an index of refraction of $1.95-0.79 i$ was used for all wavelengths, based on the work of Bond and Bergstrom (2006). This high absorption value represents a limiting case that is more relevant for primary emitted spherules/granules $(r \sim 12.5 \mathrm{~nm})$ unlikely to be found in the upper troposphere. For smoke $(r \sim 75 \mathrm{~nm})$, which is affected by aging and coating processes in the atmosphere, we used a combination of index of refraction values obtained from AERONET observations (Holben et al., 1998) and other sources (Table 2).
The AERONET values were based on Level 2.0 data (http: //aeronet.gsfc.nasa.gov) from the station at Ilorin $\left(8.3^{\circ} \mathrm{N}\right.$, $4.3^{\circ} \mathrm{E}$ ) during strong and non-dust-contaminated biomass burning emissions on 8 January 2008. For our simulations at $813 \mathrm{~nm}$, we took the average of retrieved real and imaginary indices at $675 \mathrm{~nm}$ and $870 \mathrm{~nm}$ reported for 8 January 2008, while at $452 \mathrm{~nm}$ we applied the $440 \mathrm{~nm}$ values available from this AERONET inversion. We increased the complex index of refraction in the near-UV based on its known spectral dependence (Kirchstetter et al., 2004) and used the $390 \mathrm{~nm}$ results of Dinar et al. (2008, Table 4) in our $377 \mathrm{~nm}$ simulations. Dinar et al. (2008) worked with $\sim 100 \mathrm{~nm}$ radius particles containing variable fractions of humic-like substances (HULIS) internally mixed with ammonium sulfate (AS) and black carbon (BC); we chose the complex index of refraction corresponding to $70 \%$ HULIS, $28 \%$ AS and $2 \%$ BC. Finally, for our simulations at $337 \mathrm{~nm}$ we applied a $30 \%$ spectral increase to the $377 \mathrm{~nm}$ complex index of refraction, following the work of Jethva and Torres (2011).

The number concentrations used in our carbonaceous simulations were informed by ECHAM5-HAM model outputs presented by Zhang et al. (2010) for insoluble Aitken-sized particles $(r \sim 5-50 \mathrm{~nm})$, which do not include dust. We report simulation results that use $\rho=20 \mathrm{~cm}^{-3}$ for both particle types, and sensitivity simulations with $\rho=0.2 \mathrm{~cm}^{-3}$ and $\rho=200 \mathrm{~cm}^{-3}$. The vertical optical thickness of the carbonaceous particles is shown in Table 4. 
Table 4. Vertical extinction optical thickness in a $5 \mathrm{~km}$ layer due to all simulated species, as described in Table 2 .

\begin{tabular}{lrrrr}
\hline & $337 \mathrm{~nm}$ & $377 \mathrm{~nm}$ & $452 \mathrm{~nm}$ & $813 \mathrm{~nm}$ \\
\hline D1 or D2 & 0.01084 & 0.01092 & 0.01108 & 0.01176 \\
PS & 0.00004 & 0.00004 & 0.00003 & 0.00001 \\
SM & 0.00764 & 0.00756 & 0.00729 & 0.00520 \\
SO4 & 0.00974 & 0.00804 & 0.00572 & 0.00152 \\
ICE & 0.01090 & 0.01100 & 0.01120 & 0.01270 \\
\hline
\end{tabular}
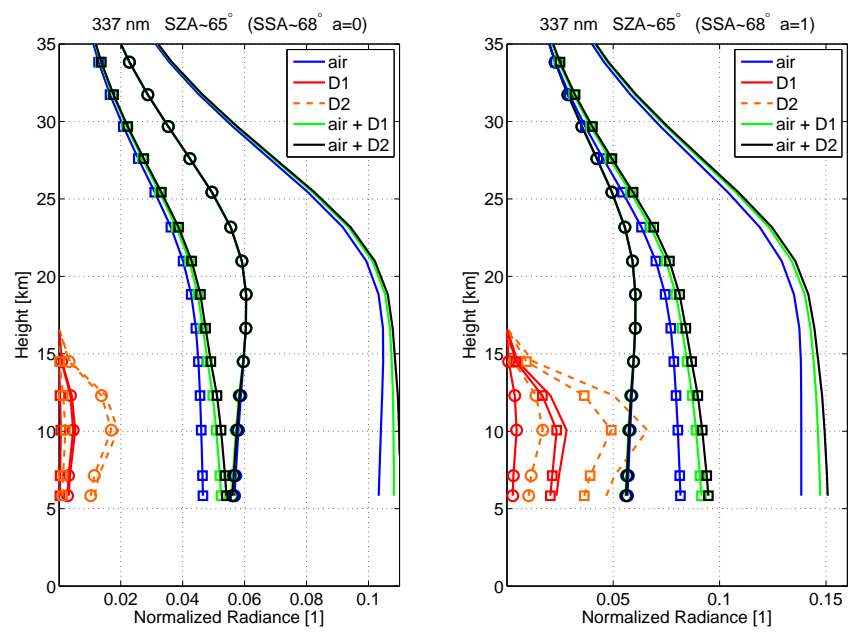

Fig. 1. Simulated limb radiance [normalized to a solar input of 1] versus height at $337 \mathrm{~nm}$ for a surface albedo of 0 (left panel) and 1 (right panel). Color distinguishes combinations of air between 0-100 km, an absorbing dust (D1) between 10-15 km, and a nonabsorbing dust (D2) also between 10-15 km. Solid and dotted lines without markers indicate total radiance while lines with circle and square markers indicate single and multiple-scattered radiance, respectively, including absorption for D1. SZA indicates solar zenith angle. SAA indicates solar azimuth angle.

\subsection{Sulfate aerosol properties}

Sulfate aerosol particles were modeled as log-normally distributed $(r=0.08 \mu \mathrm{m}, \sigma=1.6 \mu \mathrm{m})$ based on previous studies, e.g., Bourassa et al. (2008a). More specifically we used a climatology of sulfate aerosol number concentrations compiled from OSIRIS retrievals between $25^{\circ} \mathrm{N}$ and $55^{\circ} \mathrm{N}$ as well as $70^{\circ} \mathrm{E}$ to $120^{\circ} \mathrm{W}$, i.e., Asia and Pacific, for May of a single year (2008). In the absence of reliable OSIRIS sulfate aerosol retrieval results below the $380 \mathrm{~K}$ potential temperature surface, the measurement-average sulfate aerosol profile was set to $30 \mathrm{~cm}^{-3}$ below $\sim 18 \mathrm{~km}$, for all heights in the troposphere as appropriate, i.e., $30 \mathrm{~cm}^{-3}$ in the $10-15 \mathrm{~km}$ aerosol layer region and $0 \mathrm{~cm}^{-3}$ everywhere else. This number concentration corresponds well to that presented by Zhang et al. (2010) based on ECHAM5-HAM model outputs in the accumulation soluble mode at $200 \mathrm{hPa}$ between $30^{\circ} \mathrm{N}$ and $60^{\circ} \mathrm{N}$.
Table 5. Calculated size parameters $(x=2 \pi r / \lambda)$ as a function of wavelength and particle size.

\begin{tabular}{llrrl}
\hline Particle type & Radius & $337 \mathrm{~nm}$ & $813 \mathrm{~nm}$ & Scattering type \\
\hline Air & $\sim 3 \AA$ & 0.006 & 0.002 & Rayleigh \\
Dust/Ice & $r \sim 1 \mu \mathrm{m}$ & 19 & 8 & Mie \\
Pure soot & $r \sim 12.5 \mathrm{~nm}$ & 0.2 & 0.1 & weak Mie \\
Smoke & $r \sim 75 \mathrm{~nm}$ & 1.40 & 0.58 & Mie \\
Sulfate aerosol & $r \sim 80 \mathrm{~nm}$ & 1.49 & 0.62 & Mie \\
\hline
\end{tabular}

The sulfate aerosol ( $75 \mathrm{wt}$. \% solution) index of refraction is that built into SASKTRAN and based on the work of Palmer and Williams (1975) for wavelengths above $360 \mathrm{~nm}$ and Torres et al. (1995) for wavelengths below $360 \mathrm{~nm}$. The index of refraction at the four wavelengths of our simulations is listed in Table 2, while the vertical optical thickness of our sulfate aerosols is shown in Table 4.

\subsection{Ice aerosol properties}

In nadir-viewing active remote-sensing applications, it is not always easy to distinguish dust and ice particles of a similar size because both particle types are depolarizing. While this is also the case in the long-path limb-viewing geometry, we anticipated that the absorbing properties of mineral dust may start to play a discernible role in its radiance signature. To assess the effect of mineral dust absorption, we modeled (non-absorbing) ice particles of the same size and number concentration as mineral dust aerosol, i.e., $r=1 \mu \mathrm{m}$, $\sigma=1.6 \mu \mathrm{m}, \rho=0.2 \mathrm{~cm}^{-3}$ in the $5 \mathrm{~km}$ slab from $10-15 \mathrm{~km}$ altitude. The ice index of refraction used was that built into SASKTRAN, which is detailed in Table 2, while the vertical optical thickness of these small ice particles is shown in Table 4.

\subsection{Size parameters}

Table 5 shows calculated size parameters $(x=2 \pi r / \lambda)$ corresponding to each aerosol type using the size distribution mode radius employed by the forward model calculations. For air, a particle size of $3 \AA$ was used. Scattering was classified into Rayleigh, Mie and Geometric regimes using the size parameter value of 0.002 as the transition to Rayleigh scattering, 0.2 as the transition to Mie Scattering and 2000 as the transition to Geometric scattering.

\section{Results and discussion}

Here we examine SASKTRAN radiative transfer calculations of the sensitivity of wavelength-dependent OSIRIS observations to different aerosol types. First, the results for a calculation with pure air are discussed. Figures 1 through 4 show simulated limb radiance at a given wavelength as a 

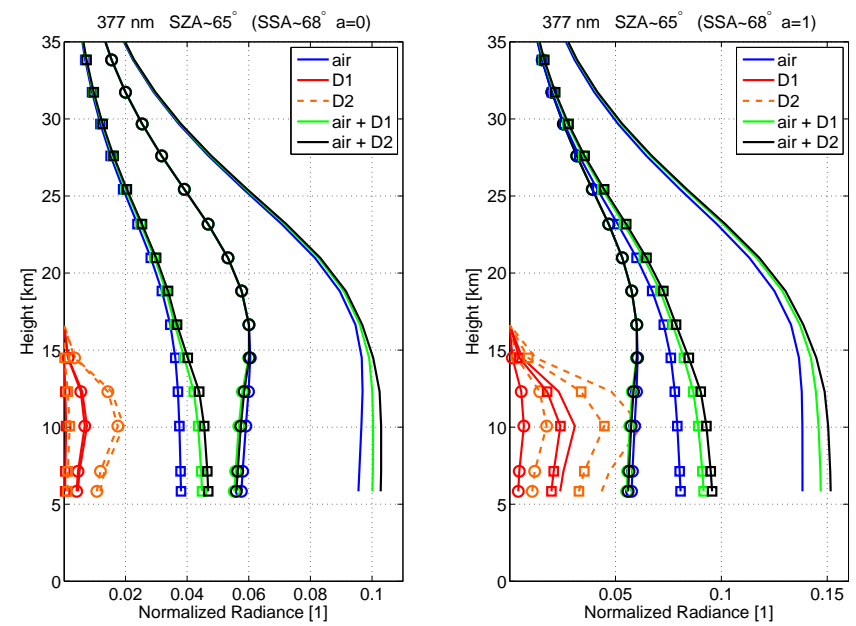

Fig. 2. As in Fig. 1 but at $377 \mathrm{~nm}$.
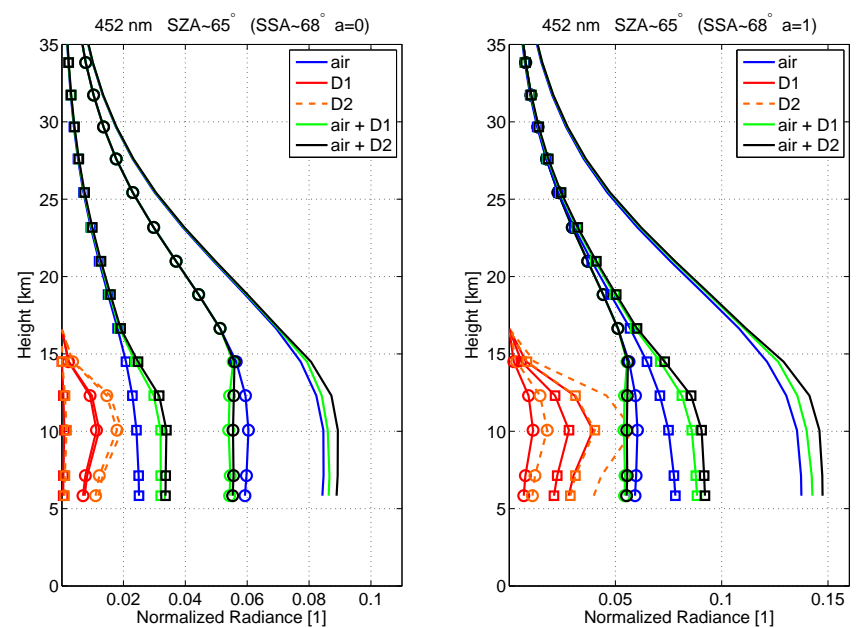

Fig. 3. As in Fig. 1 but at $452 \mathrm{~nm}$.

function of height, normalized to the input solar radiance at that wavelength. The left panel of each figure shows simulated radiances for a surface albedo of 0 while the right panel shows simulations for a surface albedo of 1 . Solid lines denote the total simulated radiance obtained through a calculation with 50 scattering orders. Lines with circle markers denote single-scattered radiances only while lines with square markers denote multiple-scattered radiances (two to 50 scattering orders). Single and multiple-scattered radiances add up to give total radiance. Line color indicates the species included in the simulation, where blue refers to air only, simulated between $0-100 \mathrm{~km}$.

\subsection{Pure air}

The single-scattered (SS) radiance of air only is identical for $a=0$ and $a=1$ at all wavelengths and heights since the surface albedo has no influence on single-scattered radiation
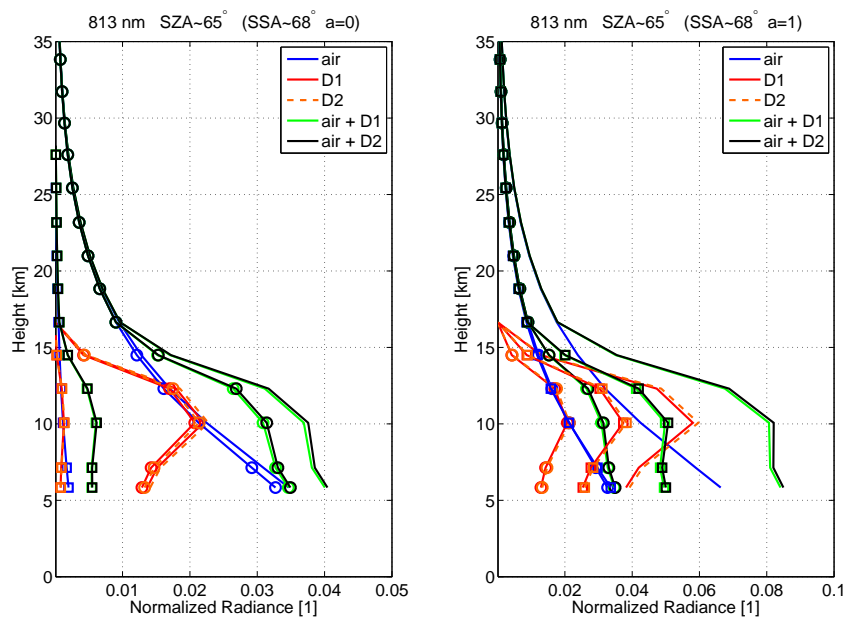

Fig. 4. As in Fig. 1 but at $813 \mathrm{~nm}$.

higher up in the atmosphere. The radiance profile increases from the top of the atmosphere until it reaches the "radiance knee", where the atmosphere becomes optically thick and below which the radiance stops increasing. As the wavelength increases, Rayleigh scattering by air molecules diminishes and the height of the radiance knee decreases from $\sim 20 \mathrm{~km}$ at $337 \mathrm{~nm}$, through $\sim 16 \mathrm{~km}$ at $377 \mathrm{~nm}$ and $\sim 11 \mathrm{~km}$ at $452 \mathrm{~nm}$ to less than $5 \mathrm{~km}$ at $813 \mathrm{~nm}$. The $10 \mathrm{~km} \mathrm{SS} \mathrm{radi-}$ ance at $813 \mathrm{~nm}$ is three times lower than at $337 \mathrm{~nm}$ due to decreased molecular scattering.

The multiple-scattered (MS) radiance of air only is smaller than the single-scattered radiance over a dark surface $(a=$ 0 , left figure panels) and decreases further from shorter to longer wavelengths, as expected. Over a bright surface ( $a=$ 1 , right figure panels) the MS radiance is much enhanced with respect to the SS radiance. At $\lambda=337,377,450 \mathrm{~nm}$ it surpasses the SS radiance, but at $\lambda=813 \mathrm{~nm}$ it is approximately equal to it.

The total radiance due to air only is the sum of single-scattered and multiple-scattered radiance.

\subsection{Mineral dust}

Figures 1 through 4 also show the sensitivity of OSIRIS to mineral dust aerosol. Dust is examined in detail because it is an important absorbing species and it is an efficient ice nucleus, thus mediating cloud formation and indirect aerosol effects on climate (e.g., Wiacek et al., 2010). Red lines in the figures refer to absorbing dust only (D1), simulated as having a non-zero number density between 10 $15 \mathrm{~km}$. Orange lines refer to non-absorbing dust only (D2), also simulated between $10-15 \mathrm{~km}$. Green refers to air plus D1, while black lines refer to air plus D2. An important general feature is that the total simulated radiance increases at all heights as the dust layer is added to the air background. While the effect of absorption is pronounced in the absence 
of air (D1 vs. D2 curves) at short wavelengths (Figs. 1 to 3 ), this difference is much reduced when dust is placed within the Rayleigh-scattered radiance field due to air molecules (air + D1 vs. air + D2 curves). Our large absorbing dust does not lead to a scene darkening (reduction in total radiance) at short wavelengths, as might be expected from the nadirviewing experience (e.g., Herman et al., 1997; Torres et al., 1998) with this aerosol species concentrated in thick plumes in the lower troposphere. The small effect of absorption is consistent with Chylek et al. (2003) who demonstrate the small sensitivity of the scattering phase function to the complex refractive index at scattering angles $<120^{\circ}$. At long wavelengths (Fig. 4), as dust absorption decreases considerably (Table 3) and the optical thickness of the Rayleigh atmosphere is also reduced, the addition of either D1 or D2 produces a pronounced increase in simulated total radiance at the dust layer height $(10-15 \mathrm{~km})$. Below, we examine the details of radiative transfer for each dust type and for the single and multiple-scattered radiance components separately.

The SS radiance of dust only (both D1 and D2) is identical for $a=0$ and $a=1$ at all wavelengths and heights, as expected. The $10 \mathrm{~km} \mathrm{SS}$ radiance of non-absorbing dust (D2) increases marginally from $337 \mathrm{~nm}$ towards $813 \mathrm{~nm}$. This is likely due to the small increase in scattering efficiency as wavelength approaches particle size, despite smearing by poly-disperse size-distribution effects. Side-scattering, at approximately $90^{\circ}$ to the direction of propagation, also increases slightly with wavelength in this observation geometry. In the limb-viewing geometry where the sun is never viewed directly, OSIRIS makes observations of solar radiance scattered by the atmosphere between $\sim 60^{\circ}$ and $\sim 120^{\circ}$. In the case of absorbing dust (D1), the $10 \mathrm{~km}$ SS radiance increases more substantially from $337 \mathrm{~nm}$ towards $813 \mathrm{~nm}$, ultimately reaching a slightly lower value as the non-absorbing dust. This is expected based on the decreasing dust absorption towards $813 \mathrm{~nm}$ and a corresponding increase (78\%) in scattering efficiency (Table 3). Figure 5 shows the phase functions for absorbing dust (D1). Side-scattering increases even more with wavelength as absorption decreases from 337 to $813 \mathrm{~nm}$. Overall, dust absorption decreases the $10 \mathrm{~km} \mathrm{SS}$ radiance by $73 \%$ at $337 \mathrm{~nm}$ (D1 with respect to D2) while the effects are small at $813 \mathrm{~nm}$, as expected.

Figures 1 to 4 also show the MS radiance of dust only (both D1 and D2), which has similar characteristics to the case of air only. The MS radiance of dust is smaller than the SS radiance over a dark surface ( $a=0$; left figure panels) and greatly enhanced with respect to the SS radiance at all wavelengths over a bright surface ( $a=1$; right figure panels). Regardless of albedo, as wavelength increases from 337 to $813 \mathrm{~nm}$, the $10 \mathrm{~km}$ MS radiance of absorbing dust (D1) increases slightly while the $10 \mathrm{~km}$ MS radiance of non-absorbing dust (D2) decreases slightly until the two are nearly equal at $813 \mathrm{~nm}$. The increase for D1 is due to a reduction in absorption while the small decrease for D2 is likely due to a net extinction of scattered radiation out of the

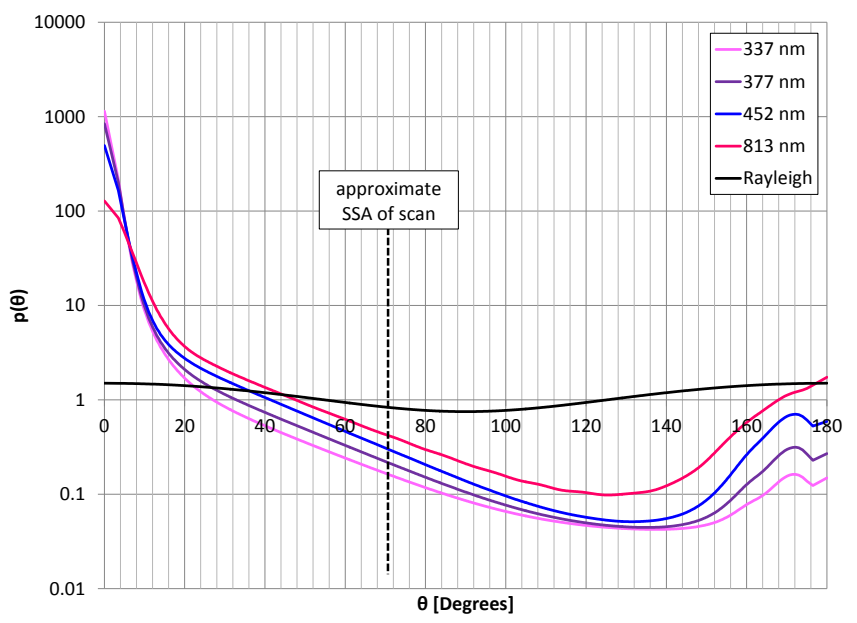

Fig. 5. SASKTRAN-calculated Mie phase functions for absorbing dust (D1) with a log-normal particle size distribution $(r=1.0 \mu \mathrm{m}$, $\sigma=1.6 \mu \mathrm{m}$ ). The Solar Scattering Angle (SSA) of the two scans in our study is also shown. At SSA $\sim 68^{\circ}$ the $813 \mathrm{~nm}$ phase function for non-absorbing dust (D2, not shown) is close to that of D1 and does not vary appreciably towards $337 \mathrm{~nm}$. Rayleigh side-scattering from $\mathrm{O}_{2}$ and $\mathrm{N}_{2}$ molecules is greater for the full range of OSIRIS observation scattering angles $\left(\sim 60^{\circ}<\mathrm{SSA}<\sim 120^{\circ}\right)$.

OSIRIS line of sight, i.e., an increase in side-scattering towards OSIRIS is more than smeared out under multiple scattering conditions. Overall, dust absorption decreases the 10$\mathrm{km}$ MS radiance by $69 \%(a=0)$ and $52 \%(a=1)$ at $337 \mathrm{~nm}$ (D1 with respect to D2) while the effects are again small at $813 \mathrm{~nm}$, as expected.

Lines with circles indicate the SS radiance of air plus dust (air $+\mathrm{D} 1)$ and air plus non-absorbing dust (air +D2). As with all other SS simulations, the SS radiances are identical for $a=0$ and $a=1$ at all wavelengths. The combination of SS radiance of air only and dust only does not add up to give the simulated SS radiance of air + dust. On the contrary, the simulated SS radiance of the air + dust combination is reduced between $10-15 \mathrm{~km}$ by both dust types at 337,377 and $452 \mathrm{~nm}$ (but not at $813 \mathrm{~nm}$, as discussed below). The biggest reduction with respect to the SS radiance of air only occurs at $452 \mathrm{~nm}$, most likely because the dust layer (spanning 10-15 km) is almost entirely above the Rayleigh radiance knee found at $\sim 11 \mathrm{~km}$. The smallest reduction is found at $337 \mathrm{~nm}$, most likely because the dust layer is found well below the knee $(\sim 20 \mathrm{~km})$. An intermediate reduction occurs at $377 \mathrm{~nm}$, when the knee is at $\sim 16 \mathrm{~km}$. Moreover, an increasing reduction with respect to air only for increasing wavelengths is opposite to the decreasing dust absorption trend with wavelength, and cannot be explained by absorption at all in the case of non-absorbing dust (D2), which exhibits the same general behavior in this respect as absorbing dust (D1). Indeed, setting dust absorption to zero only slightly offsets the SS radiance reduction, which is related primarily to where the dust layer is in relation to the knee of 

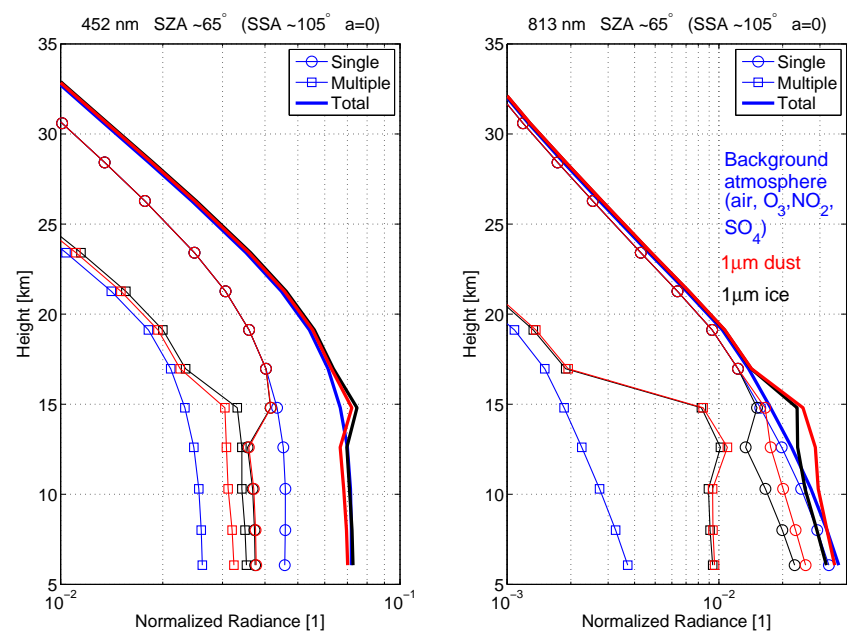

Fig. 6. The effect of a $1 \mathrm{~km}$ layer of dust (red) or ice (black) at 14$15 \mathrm{~km}$, superimposed on a background atmosphere (blue). Round markers indicate single scattering, square markers indicate multiple scattering, and thick lines indicate total scattering.

pure Rayleigh-scattered radiance. At $10 \mathrm{~km}$, absorption reduces the SS radiance of air plus dust as compared to air plus non-absorbing dust by $0.4 \%$ at $337 \mathrm{~nm}, 1.2 \%$ at $377 \mathrm{~nm}$, and $2.5 \%$ at $452 \mathrm{~nm}$, even though absorption is greater at $337 \mathrm{~nm}$ than at $452 \mathrm{~nm}$, decreasing smoothly between the two wavelengths. The absorbing characteristics of the particle type are entirely secondary. The reduction, when non-negligible, is due to the more forward-scattering (less OSIRIS-directed side-scattering) characteristics of dust particles as compared to air molecules. Scattering phase function is important for the single scattering scenario.

At $813 \mathrm{~nm}$, however, the SS radiance of the air + dust combination is increased around $10-15 \mathrm{~km}$ by both dust types, appearing more like a linear combination of air only plus dust only. At $10 \mathrm{~km}$, absorption also reduces the SS radiance of air plus dust as compared to air plus non-absorbing dust by $1.7 \%$, however, the combined effects of absorption and scattering result in an increase. While dust is also less side-scattering than air at $813 \mathrm{~nm}$ (Fig. 5), the simulations show (Fig. 4) an increasing SS radiance because the relative contribution of the Rayleigh-scattered radiance field at the altitude of the dust layer is much smaller $(1: 1)$ than at the short wavelengths (about $3: 1$ ), i.e., at $813 \mathrm{~nm}$ either dust type contributes as much as air molecules in the dust layer height in terms of the single-scattered radiance component.

Here we examine the different characteristics of dust and ice, before studying ice more closely in the context of other aerosols. Figure 6 shows a separate set of simulations at 452 and $813 \mathrm{~nm}$ for a thin $(1 \mathrm{~km})$ and dense $\left(\rho=2 \mathrm{~cm}^{-3}\right)$ dust layer situated between $14-15 \mathrm{~km}$ and superimposed on a background atmosphere containing air, $\mathrm{O}_{3}, \mathrm{NO}_{2}$ and sulfate aerosols. The figure also includes simulations for an identically setup ice layer. At both wavelengths these aerosol lay- ers are above the radiance knee. In the left panel $(452 \mathrm{~nm})$, the single-scattered radiance is reduced below the aerosol layers, slightly more so for ice than for dust, despite the fact that ice is practically non-absorbing at this wavelength while dust is still strongly absorbing. This is consistent with the reduction being caused by a directional decrease in scattered radiation and that the ice particles' phase function is slightly less side-scattering than that of dust at $452 \mathrm{~nm}$ (not shown). The effect of dust absorption is only apparent as a reduction in the multiply-scattered signal below $15 \mathrm{~km}$. In terms of the total radiance at $452 \mathrm{~nm}$, we see that both dust and ice cause an enhancement within the aerosol layer height, whereas below the layer height only dust causes an appreciable reduction in total radiance (ice causes a small total radiance reduction between $9-13 \mathrm{~km}$ ). Without a careful consideration of single and multiple scattering, it might be tempting to attribute this total radiance reduction to a more simple absorption effect instead of a directional decrease in scattered radiation.

In the right figure panel $(813 \mathrm{~nm})$ the single-scattered radiance is slightly increased above the Rayleigh background by dust while ice particles still have a decreasing effect. Below the $1 \mathrm{~km}$ aerosol layer, the single-scattered Rayleigh background is significantly reduced by ice and also to a lesser extent by dust. Given that ice absorption is still negligible at $813 \mathrm{~nm}$ compared to dust absorption, the reduction in the single-scattered Rayleigh background caused by ice is once again due to its directional scattering properties. Indeed, at $813 \mathrm{~nm}$, ice is even less side-scattering than dust as compared to at $452 \mathrm{~nm}$ (not shown). The effect of dust absorption at $813 \mathrm{~nm}$ is not strong enough to reduce the multiply-scattered radiance below that of ice at any height in the atmosphere, as was the case with a reduction below $15 \mathrm{~km}$ at $452 \mathrm{~nm}$. Again, careful consideration of single and multiple scattering, as well as relative absorption and phase function effects, are needed to explain the total radiance signature due to a mixture of air and dust or air and ice.

Returning to the simulations in Figs. 1 through 4, some general statements can be made regarding the SS radiance of the combination of air and dust (or ice). First, a dust layer well below the radiance knee has negligible effects on the SS Rayleigh radiance field. Second, a dust layer around the height of the radiance knee shields the SS Rayleigh radiance field at and below the dust layer. Third, a dust layer well above the radiance knee compares with or dominates the SS Rayleigh radiance field. The extent to which the SS Rayleigh radiance shielding effect competes with the increased direct (i.e., single-) scattering effect is the extent to which combining the molecular atmosphere with dust particles is nonlinear, i.e., non-additive. This interpretation only becomes clear when the effect of dust absorption is explicitly disentangled in the simulations presented in Figs. 1 through 4. Moreover, Fig. 6 shows that the effect is very similar for ice, even though there is a significant difference between dust 

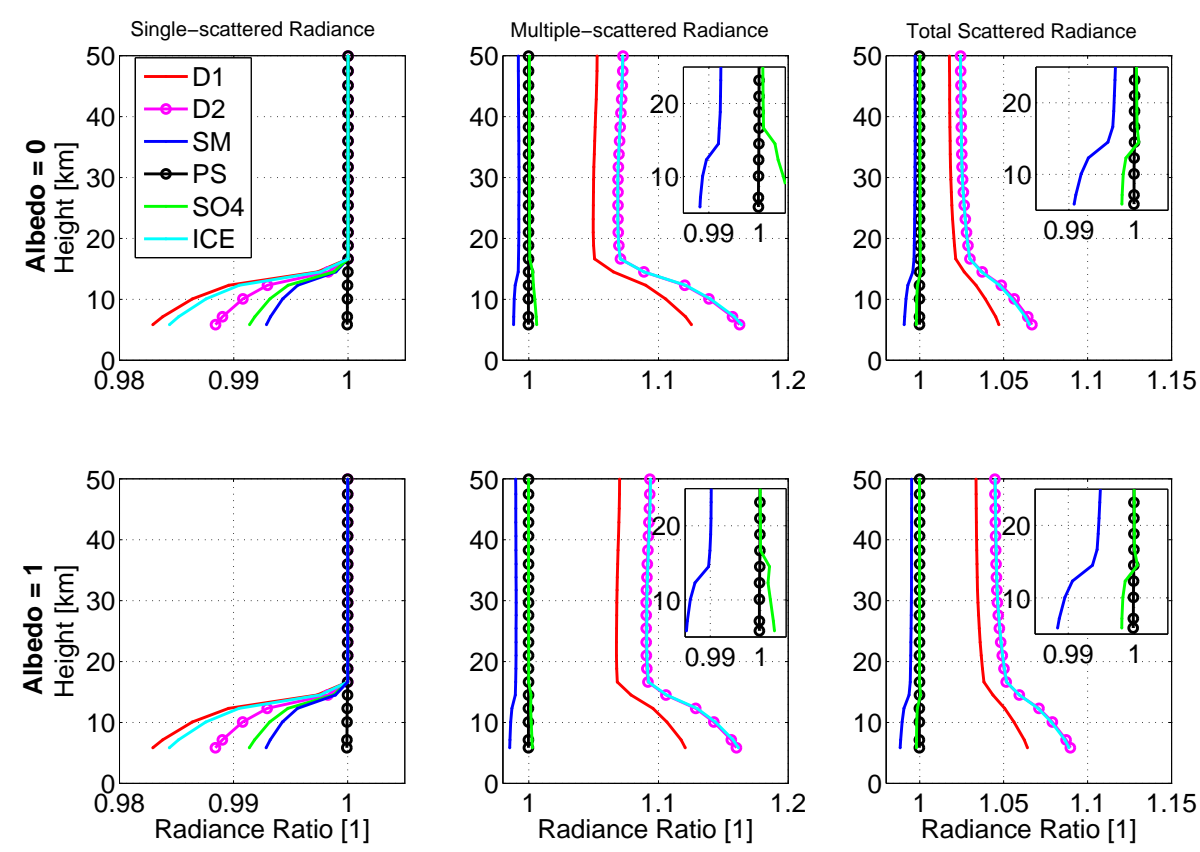

Fig. 7. Simulations of radiance due to air plus a given aerosol species divided by radiance due to air only at $337 \mathrm{~nm}$. Species details are in Table 2. The left column shows single-scattered radiance, the middle column shows multiple-scattered radiance, and the right column shows total scattered radiance, as viewed by OSIRIS for SZA $\sim 65^{\circ}$ and for SAA $\sim 70^{\circ}$. The top row shows simulations for an albedo of 0 while the bottom row shows simulations for an albedo of 1 .

and ice particles' absorbing properties. Overall, the absorption effects of dust are small in this viewing geometry.

Finally, we analyze the MS radiance of air plus dust (air + D1) and air plus non-absorbing dust (air + D2). Except at the longest wavelength (Fig. 4), the mixture behaves more closely to pure air than to either of the pure dust cases. Moreover, like in the case of MS radiance in pure air, the MS radiance of the air-dust mixtures is lower than SS radiance over a dark surface and it is higher than SS radiance over a bright surface. Unlike the spatially confined effect of air-dust mixtures on SS radiance (15 km and below), the effect on MS radiance extends throughout the atmosphere $(0-100 \mathrm{~km})$. The effect of dust absorption, i.e., the difference between air plus dust and air plus non-absorbing dust curves is small at the shorter wavelengths and nearly negligible at $813 \mathrm{~nm}$, where dust absorption is also much decreased. Most importantly, whether or not the dust is absorbing, the MS radiance of the air plus dust mixture is always higher than the MS radiance of air only, with the air plus non-absorbing dust mixture yielding the highest MS radiance, as expected. It is this increased MS radiance that is responsible for an increased total (MS + SS) radiance at all heights (discussed above), and an overall brighter scene, regardless of dust absorbing properties. We note that these conclusions and the general appearance of Figs. 1 through 4 are not influenced by particle nonsphericity. This occurs because non-spherical and spherical scattering phase functions are similar (within $8-17 \%$ in our case) at solar scattering angles $<90^{\circ}$, which leads to a neg- ligible effect $(<1 \%)$ on the single-scattered radiances. The multiple-scattered and thus also the total scattered radiances are increased within and above the non-spherical dust particles layers, leading to similar but stronger scene brightening effects.

\subsection{Other aerosols: carbonaceous, sulfate and small ice particles}

To understand the effect of aerosols in the limb-scatter viewing geometry more fully, we performed simulations with two types of absorbing carbonaceous particles and non-absorbing sulfate aerosol particles for the same simulation conditions of observation geometry and layer thickness as in the case of mineral dust. Small ice particles were also simulated (same size, number concentration and vertical layer placement as mineral dust), since dust and ice are often hard to distinguish in remote-sensing measurements (e.g., Omar, 2009).

Figure 7 shows simulations of radiance due to air plus a given aerosol species, divided by radiance due to air only at $337 \mathrm{~nm}$. The left column shows single-scattered (SS) radiance; the middle column shows multiple-scattered (MS) radiance, and the right column shows total scattered radiance, as viewed by OSIRIS for the observation geometry of $\mathrm{SZA} \sim 65^{\circ}$ and $\mathrm{SAA} \sim 70^{\circ}$. The top row shows simulations for an albedo of 0 while the bottom row shows simulations for an albedo of 1 . Figures 8, 9 and 10 are arranged in the same way, showing simulations for 377,452 and $813 \mathrm{~nm}$, respectively. At all wavelengths, the single-scattered radiance 

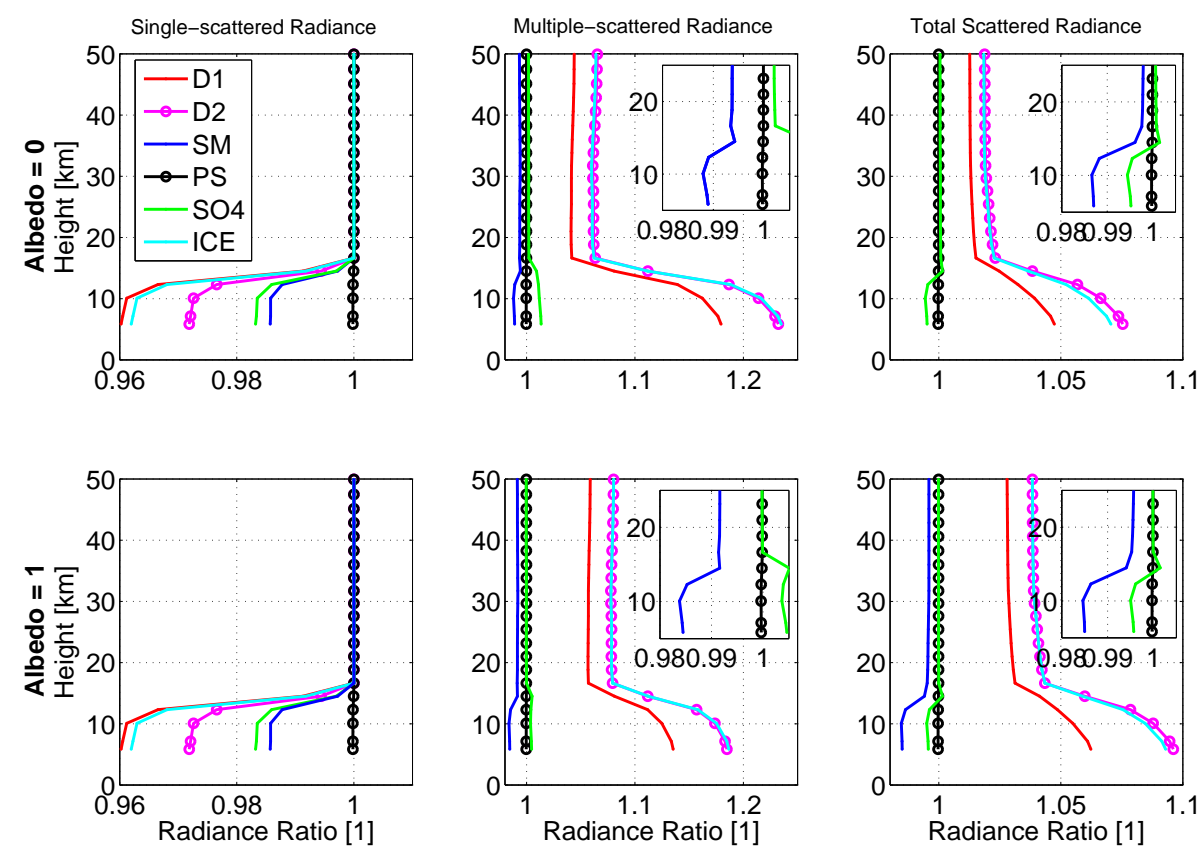

Fig. 8. As in Fig. 7 but at $377 \mathrm{~nm}$.

(left column) is unaffected by surface albedo (top vs. bottom row), as expected and as discussed previously for mineral dust. At short wavelengths, all aerosol species included in our simulations, regardless of absorption, act to reduce the amount of single-scattered radiation reaching OSIRIS as compared to the situation of having purely Rayleighscattering air only. This is consistent with our previous discussion of mineral dust single scattering. Moreover, the most absorbing aerosol species (pure soot) has the least effect on the single-scattered radiance field of pure air (the pure soot radiance ratio is nearly coincident with 1 at all heights in the left column of Figs. 7 through 10), on account of its smallest size and least scattering efficiency. Pure soot, smoke and sulfate aerosols are simulated at similar and realistic number concentrations of 20,20 and $30 \mathrm{~cm}^{-3}$, respectively. Their sizes, however, range from a mode radius of $12.5 \mathrm{~nm}$ for pure soot, through $75 \mathrm{~nm}$ for smoke, to $80 \mathrm{~nm}$ for sulfate aerosol. Clearly size, phase function and number concentration determine the dominant single-scattering radiance reduction effects, not particle absorption, with the largest reduction in single-scattered radiance among these three aerosol types in fact resulting from the least absorbing sulfate aerosol particles (likely on account of their highest number concentration, as compared to the relatively more absorbing but fewer smoke particles of a very similar size). Among the three large aerosol types (D1, D2 and ICE), which are simulated at exactly the same size and number concentration, the largest reduction in single-scattered radiance is due to absorbing dust, however, the effect of negligibly absorbing ice particles is similar, showing the importance of phase function in single-scattering. Non-absorbing dust results in the smallest reduction of the single-scattered radiance among the three large aerosol types. At 377 and $452 \mathrm{~nm}$, as the radiance knee shifts below the aerosol layer, the effect of the aerosols remains qualitatively similar, but becomes larger. By $452 \mathrm{~nm}$, the effect of the aerosol layer becomes more coincident with its vertical extent (10-15 km). As the absorption of D1 decreases towards $452 \mathrm{~nm}$, ice overtakes D1 as the aerosol particle resulting in the greatest reduction in single-scattered radiance as compared to pure air. By $813 \mathrm{~nm}$, as the single-scattered radiance of pure air decreases significantly, all the aerosol particles except pure soot lead to an increase in the single-scattered radiance observed by OSIRIS within the aerosol layer, which is consistent with our previous discussion of single-scattering of mineral dust.

The middle columns of Figs. 7 through 10 show the simulated ratio of the MS radiance due to an aerosol layer plus air to the MS radiance due to air only. The results for an albedo of 0 (top row) are qualitatively similar to those for an albedo of 1 (bottom row) at all wavelengths except that over a dark surface the radiance ratios are higher. This occurs because in the absence of strong Rayleigh multiple scattering over a dark surface, the multiple scattering due to the aerosol component represents a higher proportion of the total MS radiance; the effect also becomes more pronounced towards longer wavelengths, as Rayleigh scattering weakens.

The addition of large and efficiently scattering particles (D1, D2, ICE) causes an unambiguous MS signal increase within and below the aerosol layer, but also above it, too (middle columns). The total (net) radiance signal detected by OSIRIS for these large particles (right columns) is enhanced 

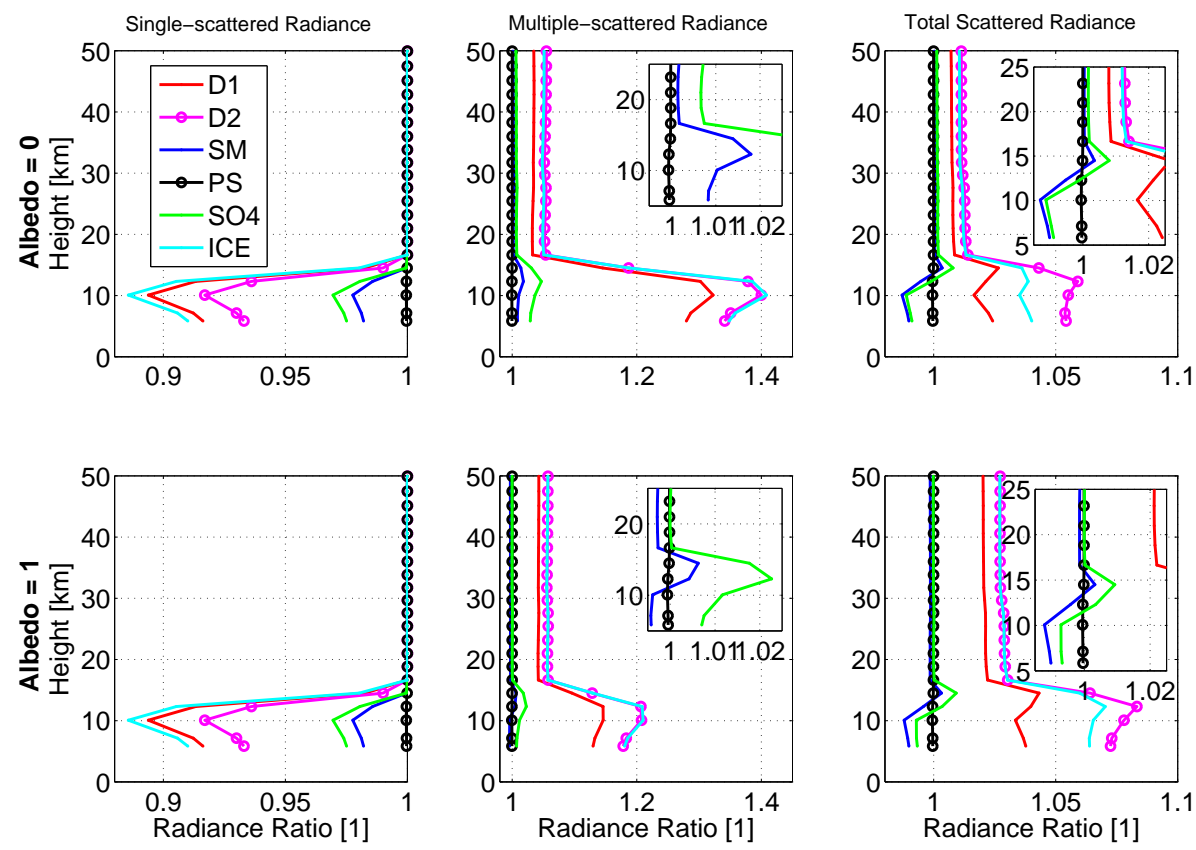

Fig. 9. As in Fig. 7 but at $452 \mathrm{~nm}$.

with respect to air only (radiance ratio $>1$ ) at all heights, despite the reductions in single-scattered signals.

MS signals from the smallest and least efficiently scattering pure soot carbonaceous particles are the smallest $(<0.1 \%)$, always attenuated with respect to air only (radiance ratio $<1)$, most noticeable at or below the soot layer and almost negligible above it (middle column insets). As such, the net radiance signals are correspondingly small and negative at all wavelengths (right column insets).

Medium-sized absorbing particles (smoke) and mediumsized non-absorbing particles (sulfate aerosol) produce medium-sized effects on MS signals at short wavelengths (middle column insets), with one key difference between the two species. Whereas scattering sulfate aerosol particles cause an increase in MS signal within, below and - to a much lesser extent - above the aerosol layer, scattering and absorbing smoke particles can do both. For example, at $452 \mathrm{~nm}$ they cause an increase in MS signal within the layer that is in part offset by a decrease at all heights due to absorption effects (Fig. 9, $a=1$ ). The particles' absorption effects above the aerosol layer (up to the top of the atmosphere) are greatest at the shortest wavelength $(337 \mathrm{~nm})$ and for the greatest surface albedo $(a=1)$, when multiple scattering effects involving the medium-sized carbonaceous particles are the greatest. However, the particles' absorption effects within and below the aerosol layer are actually greatest at $377 \mathrm{~nm}$, where absorption is still high, but the radiance knee $(\sim 17.5 \mathrm{~km})$ is not far above the top of the aerosol layer $(15 \mathrm{~km})$. At a longer wavelength $(452 \mathrm{~nm})$ the absorption effects above and below the layer are visible at an albedo of 1 (as a decrease in the radiance ratio to values $<1$ in MS signal), but no longer at an albedo of 0 .

The total (net) radiance signal for sulfate aerosol particles (right column insets) exhibits an increase above air only levels at the top of the sulfate aerosol layer and a decrease below air only levels within and below the sulfate aerosol layer. The total (net) radiance signal for smoke particles is similar in shape and magnitude to that of sulfate aerosol at $452 \mathrm{~nm}$ (right column insets), where the particles' absorption effects are least amplified due to a weaker multiple scattering effect. At the shortest wavelength $(337 \mathrm{~nm}$, right column insets), the total radiance ratio of medium-sized carbonaceous particles is always attenuated (radiance ratio $<1$ ) with respect to air only levels since the particles' absorption effects are now more amplified due to a stronger multiple-scattering effect. There is more attenuation within an below the smoke layer than within. It is clear that the multiple-scattering effects within the air layer itself do not amplify aerosol absorption because if that were the case then the pure soot particles would produce strong absorption signals. Finally, at long wavelengths $(813 \mathrm{~nm})$ both medium-sized aerosol particles (smoke and sulfate aerosol) produce very similar MS signals due to their similar scattering effects. The MS and total (net) radiance ratios are $>1$ at all heights for both species.

In summary, the presence of absorbing medium-sized particles (e.g., smoke) can in theory be discerned at the shortest wavelengths using the fact that they create a small $(\sim 1 \%)$ attenuation in signal with respect to air only (radiance ratio $<1)$ in the entire atmosphere above the aerosol layer, originating from the MS radiance component. No other aerosol type in our study has this signal. However, attenuated total 

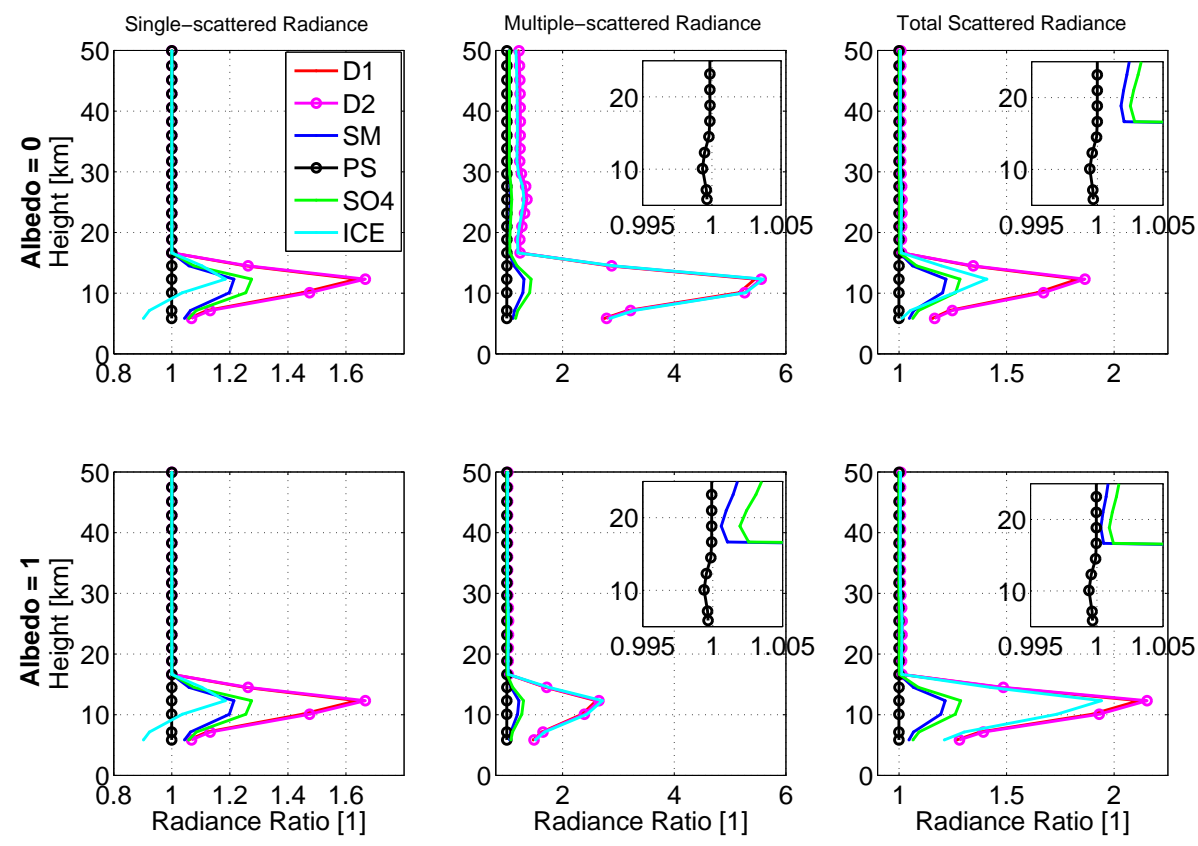

Fig. 10. As in Fig. 7 but at $813 \mathrm{~nm}$. D2 obscures D1 almost completely due to low D1 absorption at $813 \mathrm{~nm}$.

radiance signals at and below a layer of absorbing mediumsized particles are due to the combination of a reduction in SS radiances through scattering effects and a reduction in MS radiances through absorption effects. The already small MS radiance reduction can vanish completely or even turn to a small radiance increase if the complex index of refraction at near-UV wavelengths is reduced (calculations for this type of aerosol are shown in the discussion version of this paper). Moreover, an attenuation in total radiance can result from the presence of sulfate aerosol.

\subsection{Sensitivity to carbonaceous aerosol}

Overall, the addition of a moderately absorbing scatterer, i.e., dust, results in increased radiances at all heights due to increased multiple scattering effects, even at the shortest wavelengths, where dust absorption is high. This is why even at $35 \mathrm{~km}$ the scene appears brighter with dust in it than without. In this section, we examine the effect of medium-sized smoke aerosols and small-sized pure soot aerosols on total OSIRIS radiances for various combinations of carbonaceous particle size, absorption, number concentration and surface albedo. Table 6 shows the percent difference at $\sim 35 \mathrm{~km}$ altitude between a simulation including only air and air plus carbonaceous aerosols in a $5 \mathrm{~km}$ layer extending from 10 to $15 \mathrm{~km}$. Negative values mean that the introduction of pure soot has reduced the radiance at $\sim 35 \mathrm{~km}$ altitude with respect to the case of air only. The scene darkening effect is more pronounced for an albedo of 1 . The percent increase or decrease in radiance as compared to pure air conditions is linear with particle number concentration. That is, once a given index of refraction, particle size and surface brightness determine the sign of the particle's effect at $\sim 35 \mathrm{~km}$, number concentration is only a scaling factor, even up to $\rho=200 \mathrm{~cm}^{-3}$.

A scene darkening at $\sim 35 \mathrm{~km}$ of up to $-4.54 \%(337 \mathrm{~nm})$ and $-0.38 \%(813 \mathrm{~nm})$ results for smoke particles over a bright surface and under extremely high particle number concentrations $\left(\rho=200 \mathrm{~cm}^{-3}\right)$. Otherwise, for more realistic number concentrations of the same particle type, e.g., $\rho=20 \mathrm{~cm}^{-3}$, we get roughly $10 \times$ smaller scene darkening of only $-0.49 \%(337 \mathrm{~nm})$ and $-0.04 \%(813 \mathrm{~nm})$. This is comparable to the OSIRIS measurement precision, which is approximately $0.1 \%$ at $337 \mathrm{~nm}$ and $1 \%$ at $813 \mathrm{~nm}$. Small and strongly absorbing pure soot particles produce a negligible scene darkening of less than $-0.09 \%(337 \mathrm{~nm})$ and $-0.04 \%$ $(813 \mathrm{~nm})$ over all ranges of number concentrations and albedos used in the simulations.

We also calculated the limiting case of a scene darkening at $\sim 35 \mathrm{~km}$ for hypothetical "hybrid" particles with a size corresponding to smoke and an absorption corresponding to pure soot. For the highest number concentration, this lead to a scene darkening of $-4.5 \%(a=0)$ and $-8.2 \%(a=1)$ at $337 \mathrm{~nm}$. At $813 \mathrm{~nm}$, there was a scene brightening of $1.2 \%$ over a dark surface $(a=0)$ and a scene darkening of $-7.7 \%$ over a bright surface $(a=1)$. At a number of concentration of $\rho=20 \mathrm{~cm}^{-3}$, the scene darkening was reduced to $-0.5 \%$ $(a=0)$ and $-0.9 \%(a=1)$ at $337 \mathrm{~nm}$. At $813 \mathrm{~nm}$, the effect was a brightening of $0.1 \%(a=0)$ and a darkening of $-0.9 \%(a=1)$. 

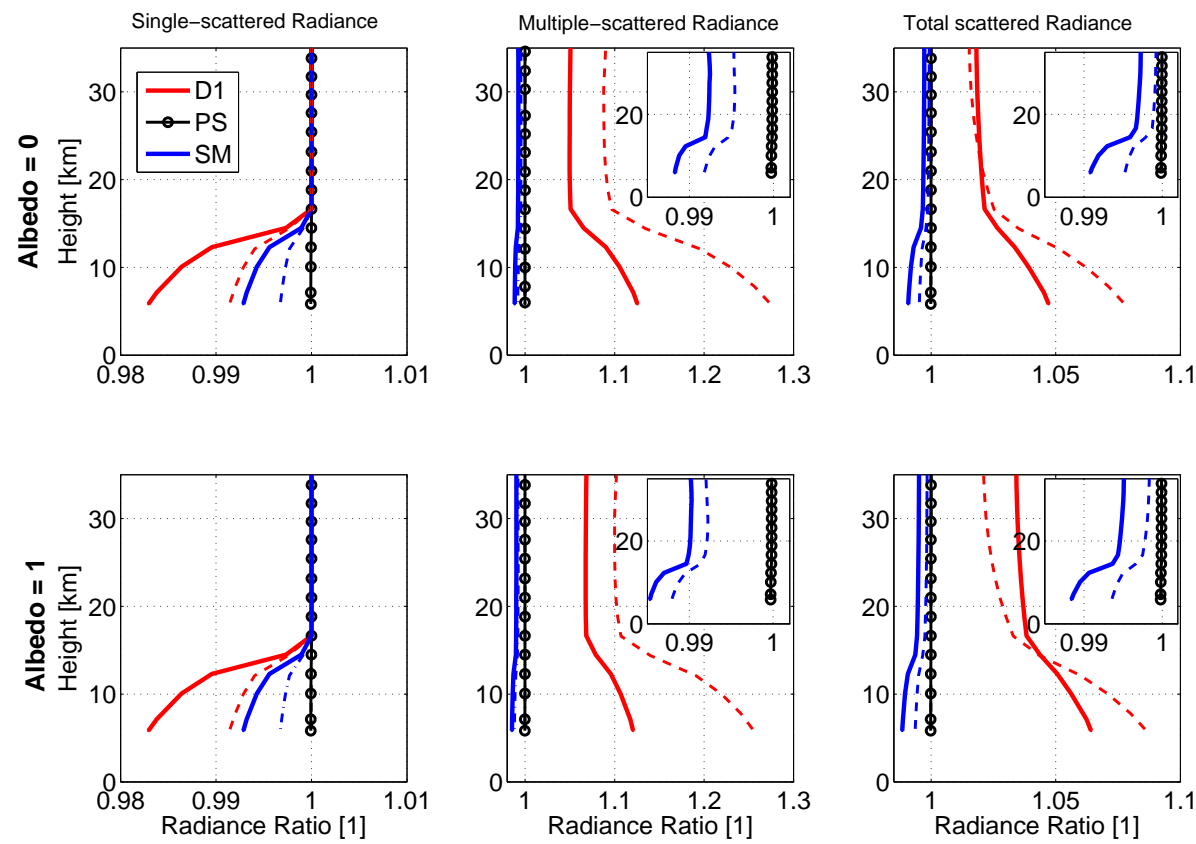

Fig. 11. A comparison of single-scattered, multiple-scattered and total scattered radiance at $337 \mathrm{~nm}$ for absorbing dust (D1), pure soot (PS) and smoke (SM) at solar zenith angles of $65^{\circ}$ (thick solid lines) and $87^{\circ}$ (thinmer dashed lines). PS for low SZA is also distinguished with circle markers and completely obscures PS for high SZA.

\subsection{Effect of SZA on mineral dust and smoke}

The limb-viewing radiance simulations described up to this point were performed at an SZA of $\sim 65^{\circ}$. In this section, we report the effect of increasing the SZA to $\sim 87^{\circ}$ on simulations of mineral dust (D1) and smoke.

At the shortest wavelength $(337 \mathrm{~nm})$ and over a dark surface $(a=0)$, a higher SZA decreased absolute levels of single-scattered (SS), multiple-scattered (MS), and total scattered (TS) radiances observed by OSIRIS for air plus dust, especially below the knee at $\sim 20 \mathrm{~km}$. However, dust absorption was not amplified appreciably by the longer path length (as compared to dust scattering) and dust still caused an increased MS and TS radiance as compared to that of air alone, as in the case of low SZA. The relative radiance levels of the air plus dust mixture to that of an air only atmosphere were in fact higher at the higher SZA for TS radiance below $15 \mathrm{~km}$. This occurred because the addition of dust increased the net scattered signal levels detected by OSIRIS (Fig. 11). For the air plus smoke mixture at $337 \mathrm{~nm}$ and over a dark surface $(a=0)$ a higher SZA also decreased absolute levels of SS, MS, and TS radiances. As in the case of dust, smoke absorption was not amplified appreciably by the longer path length (as compared to smoke scattering) and the addition of smoke still caused just a slight decrease in TS radiance as compared to that of air alone, similar to the case of low SZA at all heights in the atmosphere. The highly-scattering high SZA conditions were not quite sufficient to increase the scattering effects to dominate over increased absorption effects, however, this can be achieved with a lower complex index of refraction for the smoke particles, as scattering and absorption are in a delicate balance in this size and absorption regime. The TS radiance reduction at all heights above the smoke layer was $\sim 0.08 \%$ (Fig. 11). The results at 377 and $452 \mathrm{~nm}$ were qualitatively similar to those at $337 \mathrm{~nm}$, with the radiance knee now found at $\sim 17.5 \mathrm{~km}$ and $\sim 12.5 \mathrm{~km}$.

At the shortest wavelength $(337 \mathrm{~nm})$ and over a bright surface $(a=1)$, the results for an air plus dust mixture were similar to results over a dark surface. This is likely because the effect of the surface (and its albedo) is low due to the atmosphere's high optical thickness. For the air plus smoke mixture at high SZA the tendency of absorption effects to dominate over scattering effects was similar to that over a dark surface, and a small TS radiance reduction $(<0.15 \%)$ was still present at all heights above the smoke layer (Fig. 11). The results at 377 and $452 \mathrm{~nm}$ were again similar to those at $337 \mathrm{~nm}$.

At the longest wavelength $(813 \mathrm{~nm})$ and over a dark surface $(a=0)$ the atmosphere is still optically thin at the high SZA, with a knee somewhere below $5 \mathrm{~km}$. For the air plus dust mixture, the relative amount of SS radiance (relative to air only) was very similar at low and high SZA; the relative amount of MS radiance (again, relative to air only) was higher at high SZA, with the net result that the relative amount of TS radiance (again, relative to air only) was only slightly higher at high SZA because MS radiance is not very significant at $813 \mathrm{~nm}$. In the case of the air plus smoke mixture, the relative amount of SS radiance was slightly higher 
Table 6. Percent difference (at $35 \mathrm{~km}$ altitude) between simulations including only air vs. air plus carbonaceous aerosols in a $5 \mathrm{~km}$ layer extending from 10-15 km. Pure soot and smoke particles are described in Table 2, while hybrid particles have the absorption of pure soot and the size of smoke, representing a limiting case of the effect of carbonaceous aerosols in limb-scatter observations.

\begin{tabular}{|c|c|c|c|c|c|c|c|c|c|c|c|c|}
\hline \multirow[b]{3}{*}{$\rho\left[\mathrm{cm}^{-3}\right]$} & \multicolumn{6}{|c|}{$a=0$} & \multicolumn{6}{|c|}{$a=1$} \\
\hline & \multicolumn{2}{|c|}{ Pure soot } & \multicolumn{2}{|c|}{ Smoke } & \multicolumn{2}{|c|}{ Hybrid } & \multicolumn{2}{|c|}{ Pure Soot } & \multicolumn{2}{|c|}{ Smoke } & \multicolumn{2}{|c|}{ Hybrid } \\
\hline & $337 \mathrm{~nm}$ & $813 \mathrm{~nm}$ & $337 \mathrm{~nm}$ & $813 \mathrm{~nm}$ & $337 \mathrm{~nm}$ & $813 \mathrm{~nm}$ & $337 \mathrm{~nm}$ & $813 \mathrm{~nm}$ & $337 \mathrm{~nm}$ & $813 \mathrm{~nm}$ & $337 \mathrm{~nm}$ & $813 \mathrm{~nm}$ \\
\hline 0.2 & -0.0001 & 0.0000 & -0.0028 & 0.0012 & -0.0054 & 0.0014 & -0.0001 & -0.0001 & -0.0049 & -0.0004 & -0.0096 & -0.0087 \\
\hline 20 & -0.0050 & -0.0003 & -0.2746 & 0.1160 & -0.5291 & 0.1366 & -0.0089 & -0.0035 & -0.4895 & -0.0402 & -0.9430 & -0.8604 \\
\hline 200 & -0.0500 & -0.0027 & -2.4633 & 1.1466 & -4.4902 & 1.1967 & -0.0884 & -0.0350 & -4.5417 & -0.3778 & -8.1904 & -7.7199 \\
\hline
\end{tabular}

at high SZA, the relative amount of MS radiance was very similar at high SZA, and the relative amount of TS radiance was slightly higher at high SZA. At the longest wavelength $(813 \mathrm{~nm})$ and over a bright surface $(a=1)$ the results for air and dust as well as air and smoke mixtures at low and high SZA were very similar to those over a dark surface $(a=0)$.

In summary, for a wide range of aerosol models and viewing geometries, limb-scatter retrievals are not sensitive to aerosol absorption.

\section{Summary and outlook}

The limb-scatter satellite viewing geometry is well suited to detecting low-concentration aerosols in the upper troposphere due to its long observation path length $(\sim 200 \mathrm{~km})$, high vertical resolution $(\sim 1-2 \mathrm{~km})$ and good geographic coverage. We have evaluated the signature of aerosols in limb-scatter satellite observations at ultraviolet, visible and near infrared wavelengths. We used the SASKTRAN 3-D radiative transfer code to simulate OSIRIS satellite observations, paying particular attention to the effects of absorption of solar radiation by mineral dust and carbonaceous aerosols. We also investigated the signature of ice particles of the same size and number concentration as our mineral dust aerosols, as well as the signature of sulfate aerosols. The simulated aerosols were placed between $10-15 \mathrm{~km}$ in the atmosphere while air molecules, when simulated, occupied the entire model atmosphere from $0-100 \mathrm{~km}$. The absorbing aerosols were simulated at enhanced but realistic number concentrations. The simulations were performed at two albedos, $a=[0$, 1], four wavelengths, $\lambda=[337,377,452,813 \mathrm{~nm}]$, and two scan geometries, corresponding to a solar zenith angle of $65^{\circ}$ and $87^{\circ}$. We examined single, multiple, and total limbscattered radiance signatures individually. Multiple scattering was calculated as the difference of a simulation with 50 scattering orders and a single-scattering simulation.

Our simulations show that absorbing dust does not lead to a scene darkening (reduction in total radiance) at short wavelengths, as might be expected from the nadir-viewing experience (e.g., Herman et al., 1997; Torres et al., 1998) with this aerosol species in the lower troposphere. The increase in total radiance at short wavelengths is the net result of a de- crease in single-scattered (SS) radiance at and below the dust layer and an increase in multiple-scattered (MS) radiance at all heights. However, the decrease in SS radiance is primarily due to particle size affecting the direction of scattering, and not due to dust absorption effects, which we find to be small in the limb-scatter viewing geometry.

Some general statements can be made regarding the SS radiance of the combination of air and dust (or ice). First, a dust layer well below the radiance knee has negligible effects on the SS Rayleigh radiance field. Second, a dust layer around the height of the radiance knee shields the SS Rayleigh radiance field at and below the dust layer, causing a reduction. Third, a dust layer well above the radiance knee compares with or dominates the SS Rayleigh radiance field. The extent to which the SS Rayleigh radiance shielding effect competes with the increased single scattering effect is the extent to which combining the molecular atmosphere with dust particles is nonlinear, i.e., non-additive. This interpretation only becomes clear when the effect of dust absorption is explicitly disentangled. We achieved this by comparing simulations of absorbing and non-absorbing dust particles, as well as dust and ice particles of the same size. The single-scattering effect is very similar for ice, even though there is a significant difference between dust and ice particles' absorbing properties. This is because the size of the two particles is identical in our simulations.

Our simulations generalize over all particle types as follows. At long wavelengths $(813 \mathrm{~nm})$ the addition of all aerosols (except pure soot) to an air only atmosphere produced a radiance increase as compared to air only, on account of the low Rayleigh scattering in air only at $813 \mathrm{~nm}$. The radiance reduction due to soot aerosol was negligible $(<0.1 \%)$ at all heights $(0-100 \mathrm{~km})$.

At short wavelengths $(337,377,452 \mathrm{~nm})$, we found that the addition of any aerosol species to an air only atmosphere caused a decrease in single-scattered radiation in the direction of OSIRIS. The reduction was clearly related to particle size first, with absorption responsible for second-order effects only. Also at short wavelengths, multiple-scattered radiation could either increase or decrease in the presence of an aerosol species, depending both on particle size and absorption. Large scatterers (ice, mineral dust) all lead to an 
increase in multiple-scattered radiation within, below and above the aerosol layer. Small, highly absorbing pure soot particles produced a multiple-scattering response that was a very small decrease $(<0.1 \%)$ at all heights, primarily confined to within and below the soot layer. Medium-sized scatterers produced a multiple-scattering response that depended on their absorbing properties. Increased radiances were simulated as compared to air only at all short wavelengths (337, 377 and $450 \mathrm{~nm}$ ) for sulfate aerosol particles (non-absorbing) while decreased radiances were simulated for smoke particles (absorbing) at 337 and $377 \mathrm{~nm}$, where multiple scattering involving the medium-sized carbonaceous particles amplified their absorbing properties. At $452 \mathrm{~nm}$, however, this effect was attenuated and albedo-dependent.

At short wavelengths, the combined effect of single scattering decreases and multiple scattering increases led to complex total radiance signatures that could not, for the most part, unambiguously distinguish absorbing versus nonabsorbing aerosols except in the case of smoke aerosols that led to a total radiance decrease (as compared to air only) at all altitudes above the aerosol layer $(15-100 \mathrm{~km})$. This unique signature was a result of the aerosols' strong absorbing properties, non-negligible scattering efficiency, and number concentrations high enough to make multiple scattering effects due to the aerosol itself significant. Thus, we show that in the limb-scatter viewing geometry scene darkening above the aerosol layer is unambiguously due to absorption whereas scene darkening within and below the aerosol layer can simply be the result of a reduction in single-scattered radiance. Our simulations show a greater scene darkening for decreasing wavelengths, increasing surface albedo, decreasing solar zenith angle, and increasing particle number concentration, however, at $337 \mathrm{~nm}$, an albedo of 1 , a solar zenith angle of $65^{\circ}$ and an unrealistic particle number concentration of $20 \mathrm{~cm}^{-3}$ this effect does not exceed $0.5 \%$ of the total radiance due to air only, making the unique identification of medium-sized carbonaceous aerosols, i.e., smoke, difficult. Once scene darkening (or brightening) is established based on the particle's index of refraction and size as well as surface brightness, it is linear with particle number concentration over three orders of magnitude.

Without a careful consideration of single and multiple scattering, as well as absorption and phase function effects, it is not easy to explain the total radiance signature due to a mixture of air and dust, or air and some other aerosol. What seems like a total radiance reduction due to absorption is instead the result of a directional decrease in scattered radiation.

A fortuitous implication of our analysis is that limb-scatter retrievals of aerosol extinction are insensitive to external information about aerosol absorption.
Acknowledgements. This work was supported by the Canadian Foundation for Climate and Atmospheric Science (CFCAS) and by the Canadian Space Agency (CSA). We thank O. Dubovik and $\mathrm{T}$. Lapyonok for providing spheroid mixture scattering phase functions corresponding exactly to our particle size distributions and refractive index.

Edited by: O. Torres

\section{References}

Ansmann, A., Tesche, M., Althausen, D., Müller, D., Seifert, P., Freudenthaler, V., Heese, B., Wiegner, M., Pisani, G., Knippertz, P., and Dubovik, O.: Influence of Saharan dust on cloud glaciation in southern Morocco during the Saharan Mineral Dust Experiment,J. Geophys. Res., 113, D04210, doi:10.1029/2007JD008785, 2008.

Bond, C. T. and Bergstrom, R. W.: Light Absorption by Carbonaceous Particles: An Investigative Review, Aerosol Sci. Technol., 40, 27-67, 2006.

Bourassa, A. E., Degenstein, D. A., and Llewellyn, E. J.: Climatology of the subvisual cirrus clouds as seen by OSIRIS on Odin, Adv. Space Res., 36, 807-812, 2005.

Bourassa, A. E., Degenstein, D. A., Gattinger, R. L., and Llewellyn, E. J.: Stratospheric aerosol retrieval with optical spectrograph and infrared imaging system limb scatter measurements, J. Geophys. Res., 112, D10217, doi:10.1029/2006JD008079, 2007.

Bourassa, A. E., Degenstein, D. A., and Llewellyn, E. J.: Retrieval of stratospheric aerosol size information from OSIRIS limb scattered sunlight spectra, Atmos. Chem. Phys., 8, 63756380, doi:10.5194/acp-8-6375-2008, 2008a.

Bourassa, A. E., Degenstein, D. A., and Llewellyn, E. J.: SASKTRAN: A spherical geometry radiative transfer code for efficient estimation of limb scattered sunlight, J. Quant. Spectrosc. Ra., 109, 52-73, 2008b.

Bourassa, A. E., Degenstein, D. A., Elash, B. J., and Llewellyn, E. J.: Evolution of the stratospheric aerosol enhancement following the eruptions of Okmok and Kasatochi: OdinOSIRIS measurements, J. Geophys. Res., 115, D00L03, doi:10.1029/2009JD013274, 2010.

Bourassa, A. E., McLinden, C. A., Sioris, C. E., Brohede, S., Bathgate, A. F., Llewellyn, E. J., and Degenstein, D. A.: Fast $\mathrm{NO}_{2}$ retrievals from Odin-OSIRIS limb scatter measurements, Atmos. Meas. Tech., 4, 965-972, doi:10.5194/amt-4-965-2011, 2011.

Chylek, P., Henderson, B., and Mishchenko, M.: Satellite based retrieval of aerosol optical thickness: The effect of sun and satellite geometry, Geophys. Res. Lett., 30, 1553, doi:10.1029/2003GL016917, 2003.

Degenstein, D. A., Bourassa, A. E., Roth, C. Z., and Llewellyn, E. J.: Limb scatter ozone retrieval from 10 to $60 \mathrm{~km}$ using a multiplicative algebraic reconstruction technique, Atmos. Chem. Phys., 9, 6521-6529, doi:10.5194/acp-9-6521-2009, 2009.

DeMott, P. J., Cziczo, D. J., Prenni, A. J., Murphy, D. M., Kreidenweis, S. M., Thomson, D. S., Borys, R., and Rogers, D. C.: Measurements of the concentration and composition of nuclei for cirrus formation, Proc. Natl. Acad. Sci. USA, 100, 14655-14660, doi:10.1073/pnas.2532677100, 2003. 
Dinar, E., Abo Riziq, A., Spindler, C., Erlick, C., Kissc G., and Rudich, Y.: The complex refractive index of atmospheric and model humic-like substances (HULIS) retrieved by a cavity ring down aerosol spectrometer (CRD-AS), Faraday Discuss., 137, 279-295, doi:10.1039/b703111d, 2008.

Ernst, F., von Savigny, C., Rozanov, A., Rozanov, V., Eichmann, K.-U., Brinkhoff, L. A., Bovensmann, H., and Burrows, J. P.: Global stratospheric aerosol extinction profile retrievals from SCIAMACHY limb-scatter observations, Atmos. Meas. Tech. Discuss., 5, 5993-6035, doi:10.5194/amtd-5-5993-2012, 2012.

Haley, C. S., Brohede, S. M., Sioris, C. E., Griffioen, E., Murtagh, D. P., McDade, I. C., Eriksson, P., Llewellyn, E. J., Bazureau, A., and Goutail, F.: Retrieval of stratospheric $\mathrm{O}_{3}$ and $\mathrm{NO}_{2}$ profiles from Odin Optical Spectrograph and Infrared Imager System(OSIRIS) limb-scattered sunlight measurements, J. Geophys. Res., 109, D16303, doi:10.1029/2004JD004588, 2004.

Haywood, J. M., Jones, A., Clarisse, L., Bourassa, A., Barnes, J., Telford, P., Bellouin, N., Boucher, O., Agnew, P., Clerbaux, C., Coheur, P., Degenstein, D., and Braesicke, P.: Observations of the eruption of the Sarychev volcano and simulations using the HadGEM2 climate model, J. Geophys. Res., 115, D21212, doi:10.1029/2010JD014447, 2010.

Herman, J. R., Bhartia, P. K., Torres, O., Hsu, C., Seftor, C., and Celarier, E.: Global distribution of UV-absorbing aerosols from Nimbus 7/TOMS data, J. Geophys. Res., 102, 16911-16922, 1997.

Holben, B. N., Eck, T. F., Slutsker, I., Tanre, D., Buis, J. P., Setzer, A., Vermote, E., Reagan, J. A., Kaufman, Y. J., Nakajima, T., Lavenu, F., Jankowiak, I., and Smirnov A.: AERONET - A federated instrument network and data archive for aerosol characterization, Remote Sens. Environ., 66, 1-16, 1998.

Jethva, H. and Torres, O.: Satellite-based evidence of wavelengthdependent aerosol absorption in biomass burning smoke inferred from Ozone Monitoring Instrument, Atmos. Chem. Phys., 11, 10541-10551, doi:10.5194/acp-11-10541-2011, 2011.

Kirchstetter, T. W., Novakov, T., and Hobbs, P. V.: Evidence that the spectral dependence of light absorption by aerosols is affected by organic carbon, J. Geophys. Res., 109, D21208, doi:10.1029/2004JD004999, 2004.

Kravitz, B., Robock, A., and Bourassa, A.: Negligible climatic effects from the 2008 Okmok and Kasatochi volcanic eruptions, J. Geophys. Res., 115, D00L05, doi:10.1029/2009JD013525, 2010.

Kravitz, B., Robock, A., Bourassa, A., Deshler, T., Wu, D., Mattis, I., Finger, F., Hoffmann, A., Ritter, C., Bitar, L., Duck, T. J., and Barnes, J. E.: Simulation and observations of stratospheric aerosols from the 2009 Sarychev volcanic eruption, J. Geophys. Res., 116, D18211, doi:10.1029/2010JD015501, 2011.

McLinden, C. A., Haley, C. S., Lloyd, N. D., Hendrick, F., Rozanov, A., Sinnhuber, B.-M., Goutail, F., Degenstein, D. A., Llewellyn, E. J., Sioris, C. E., Van Roozendael, M., Pommereau, J. P., Lotz, W., and Burrows, J. P.: Odin/OSIRIS observations of stratospheric BrO: Retrieval methodology, climatology, and inferred Bry, J. Geophys. Res., 115, D15308, doi:10.1029/2009JD012488, 2010.
Müller, D., Lee, K.-H., Gasteiger, J., Tesche, M., Weinzierl, B., Kandler, K., Müller, T., Toledano, C., Otto, S., Althausen, D., and Ansmann, A.: Comparison of optical and microphysical properties of pure Saharan mineral dust observed with AERONET Sun photometer, Raman lidar, and in situ instruments during SAMUM 2006, J. Geophys. Res., 117, D07211, doi:10.1029/2011JD016825, 2012.

Müller, T., Schladitz, A., Massling, A., Kaaden, N., Kandler, K., and Wiedensohler, A.: Spectral absorption coefficients and imaginary parts of refractive indices of Saharan dust during SAMUM1, Tellus, 61B, 79-95, doi:10.1111/j.1600-0889.2008.00399.x, 2009.

Omar, A. H., Winker, D. M., Kittaka, C., Vaughan, M. A., Liu, Z., Hu, Y., Trepte, C. R., Rogers, R. R., Ferrare, R. A., Lee, K.-P., Kuehn, R. E., and Hostetler, C. A.: The CALIPSO Automated Aerosol Classification and Lidar Ratio Selection Algorithm, J. Atmos. Oceanic Technol., 26, 1994-2014, 2009.

Palmer, K. F. and Williams, D.: Optical constants of sulfuric acid: application to the clouds of Venus?, Appl. Opt., 14, 208-219, 1975.

Sokolik, I. N. and Toon, O. B.: Incorporation of mineralogical composition into models of the radiative properties of mineral aerosol from UV to IR wavelengths, J. Geophys. Res., 104, 9423-9444, 1999.

Taha, G., Rault, D. F., Loughman, R. P., Bourassa, A. E., and von Savigny, C.: SCIAMACHY stratospheric aerosol extinction profile retrieval using the OMPS/LP algorithm, Atmos. Meas. Tech., 4, 547-556, doi:10.5194/amt-4-547-2011, 2011.

Tegen, I.: Modeling the mineral dust aerosol cycle in the climate system, Quaternary Sci. Rev., 22, 1821-1834, 2003.

Torres, O., Herman, J. R., Bhartia, P. K., and Ahmad, Z.: Properties of Mount Pinatubo aerosols as derived from Nimbus 7 total ozone mapping spectrometer measurements, J. Geophys. Res., 100, 14043-14055, doi:10.1029/95JD01224, 1995.

Torres, O., Bhartia, P. K., Herman, J. R., Ahmad, Z., and Gleason, J.: Derivation of aerosol properties from satellite measurements of backscattered ultraviolet radiation: Theoretical basis, J. Geophys. Res., 103, 17099-17110, doi:10.1029/98JD00900, 1998.

Wiacek, A., Peter, T., and Lohmann, U.: The potential influence of Asian and African mineral dust on ice, mixed-phase and liquid water clouds, Atmos. Chem. Phys., 10, 8649-8667, doi:10.5194/acp-10-8649-2010, 2010.

Zhang, K., Wan, H., Wang, B., Zhang, M., Feichter, J., and Liu, X.: Tropospheric aerosol size distributions simulated by three online global aerosol models using the M7 microphysics module, Atmos. Chem. Phys., 10, 6409-6434, doi:10.5194/acp-10-64092010, 2010. 